\title{
Search for New Physics in Heavy Quark Decays at LHCb
}

\author{
Jeremy Dickens \\ on behalf of the LHCb collaboration
}

QCD2010, Montpellier 


\section{Introduction}

- Data taking at LHCb has progressed rapidly

2010/05/27 08.08

- Collected $14 \mathrm{nb}^{-1}$

- Significant strange, charm samples

- Observations of beauty

- Expect 200 pb-1 in 2010

- $\Rightarrow \mathrm{O}\left(10^{11}\right)$ bb events

- $~ 1 \mathrm{fb}^{-1}$ in 2011

- Will present in this talk:

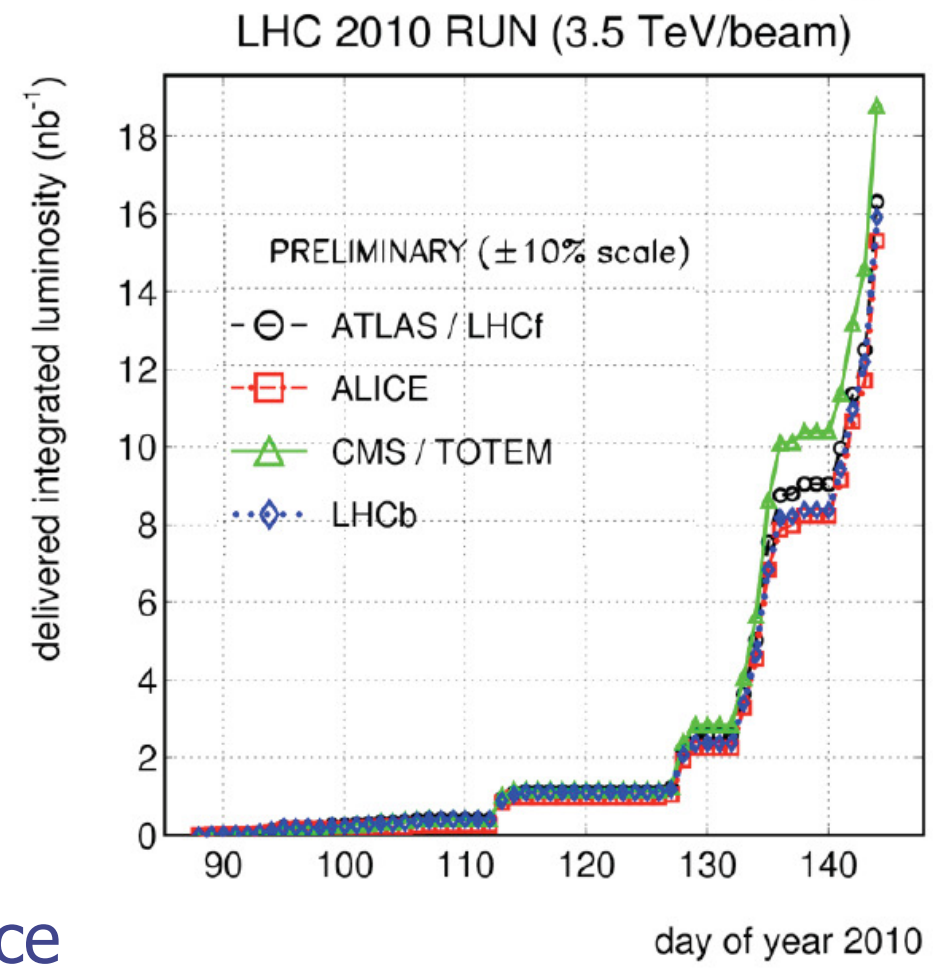

- Overview of the detector performance

- Highlights of upcoming measurements in D and B decays

- Charm: $\mathrm{y}_{\mathrm{CP}}$ in $\mathrm{D} \rightarrow \mathrm{K} \pi, \mathrm{KK}, \pi \pi$

- Beauty: $B_{s} \rightarrow \mu \mu, B_{s} \rightarrow J / \psi \phi, A_{F B}$ in $B_{d} \rightarrow K^{*} \mu \mu$ 


\section{LHCb Detector}

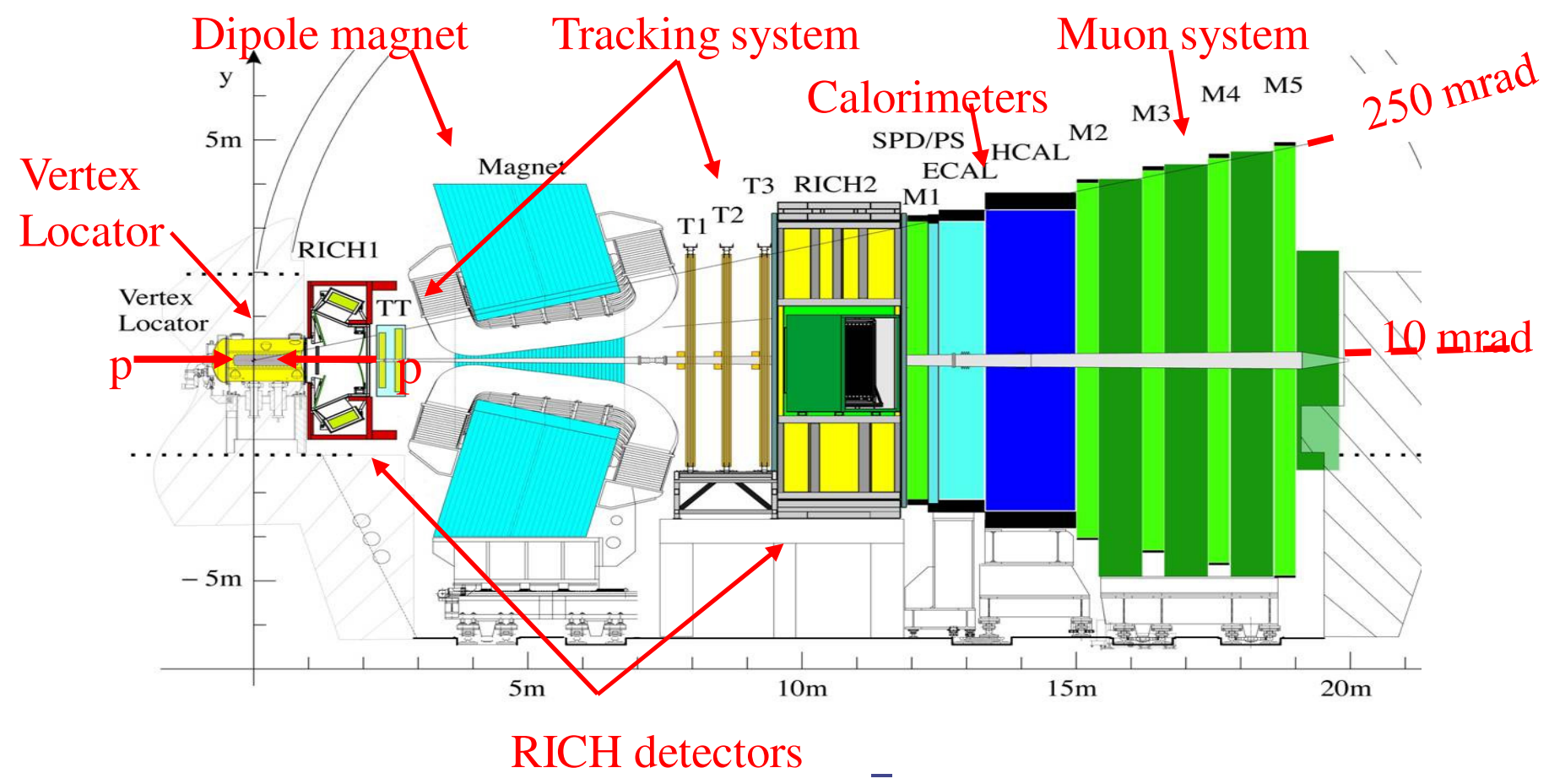

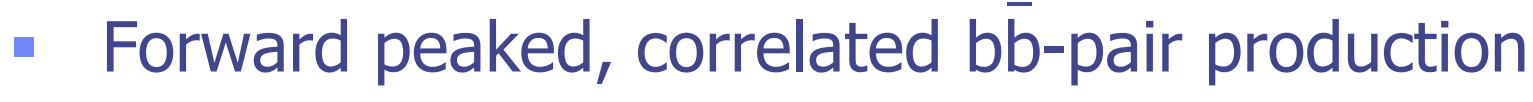

- $\mathrm{LHCb}$ is a forward spectrometer

- Sophisticated trigger system $(40 \mathrm{MHz} \rightarrow 2 \mathrm{kHz})$

- Currently run with reduced thresholds 


\section{Open Charm}

- Lower trigger thresholds $\Rightarrow$ good prospects for charm

- Large samples already collected

- Cross-section measurements

- Search for $D^{0} \rightarrow \mu \mu$

- Mixing, CPV etc
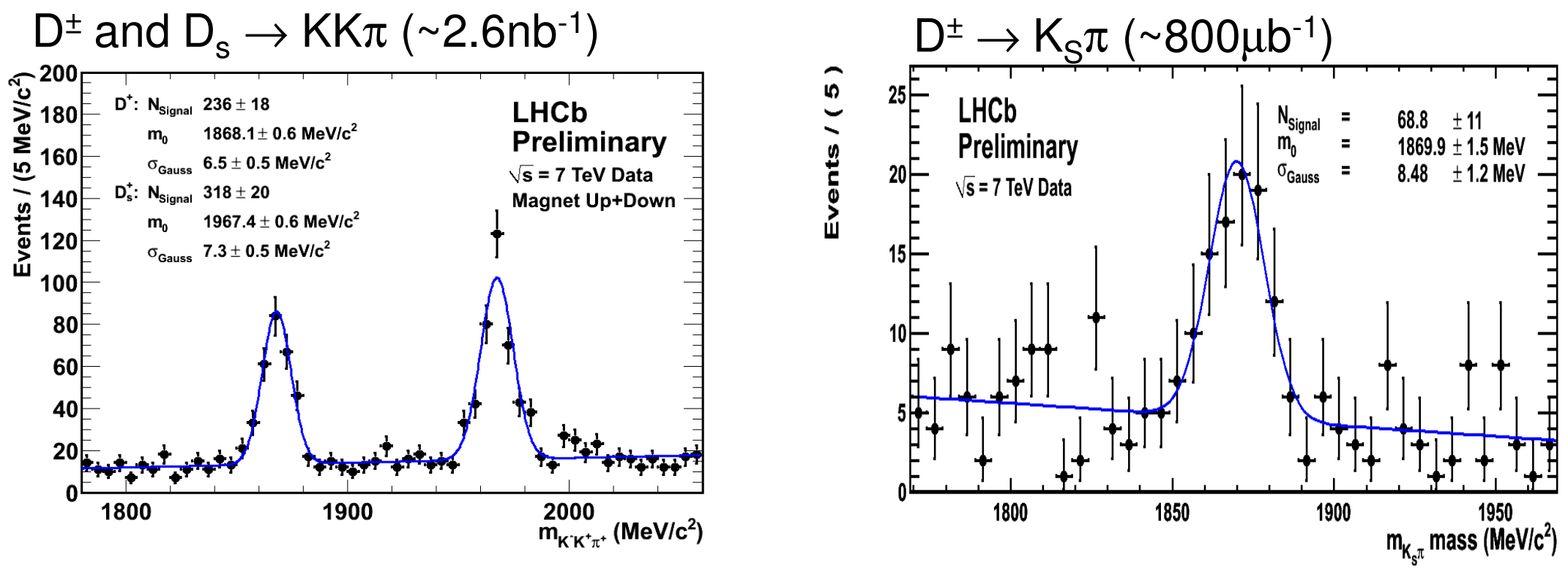


\section{Charm measurements}

- CP-violation weakly constrained

- Negligible in SM, large NP contributions possible (O(1\%) level)

- eg: direct CPV in $\mathrm{D}^{+} \rightarrow \mathrm{K}^{+} \mathrm{K}^{-} \pi^{+}\left(\mathrm{D}^{+} \rightarrow \mathrm{K}_{\mathrm{S}} \pi^{+}\right)$

- Mixing in $\mathrm{D}^{0}$ system

- No single $>5 \sigma$ observation

- Many analyses in preparation at LHCb

- For example: $\mathrm{y}_{\mathrm{CP}}$ from CP eigenstates

$$
y_{C P}=\frac{\tau\left(D^{0} \rightarrow K^{-} \pi^{+}\right)}{\tau\left(D^{0} \rightarrow K^{+} K^{-}, \pi^{+} \pi^{-}\right)}-1
$$

- Fit for event yields in bins of lifetime

- Plot ratio of yields $\rightarrow$ main systematics cancel

- Extract $\mathrm{y}_{\mathrm{CP}}$

Or extract $\mathrm{y}_{\mathrm{CP}}$ directly from the lifetime distributions
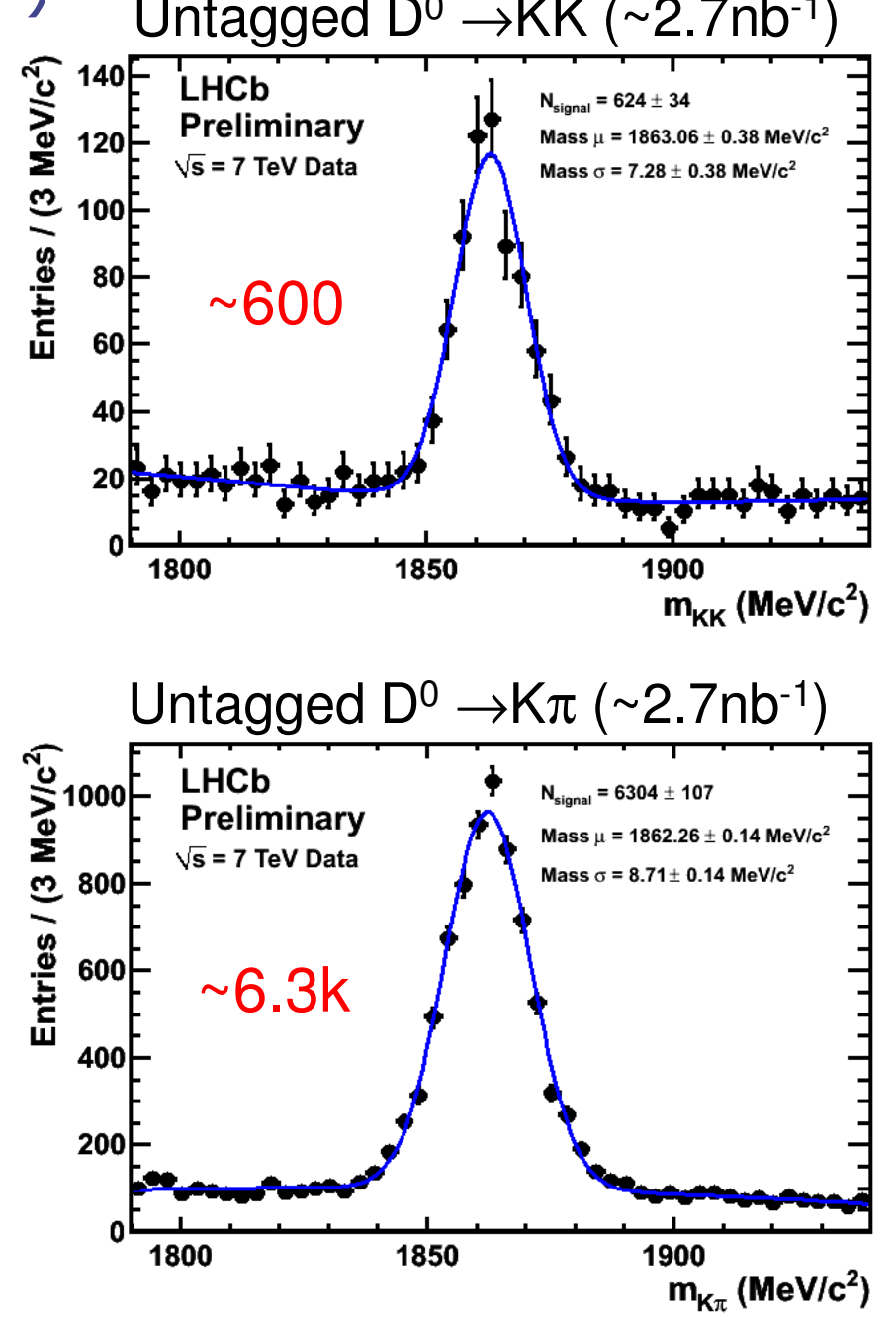


\section{$\mathrm{J} / \psi \rightarrow \mu \mu:$ Muon ID}

- $\mathrm{J} / \psi$ "tag and probe"

- $\varepsilon(\mu)=(97.3 \pm 1.2) \%$

- $\pi \rightarrow \mu$ mis-id (from $\mathrm{K}_{\mathrm{S}}$ ) in good agreement with MC

- $p \rightarrow \mu$ mis-id (from $\Lambda$ ) in good agreement with MC

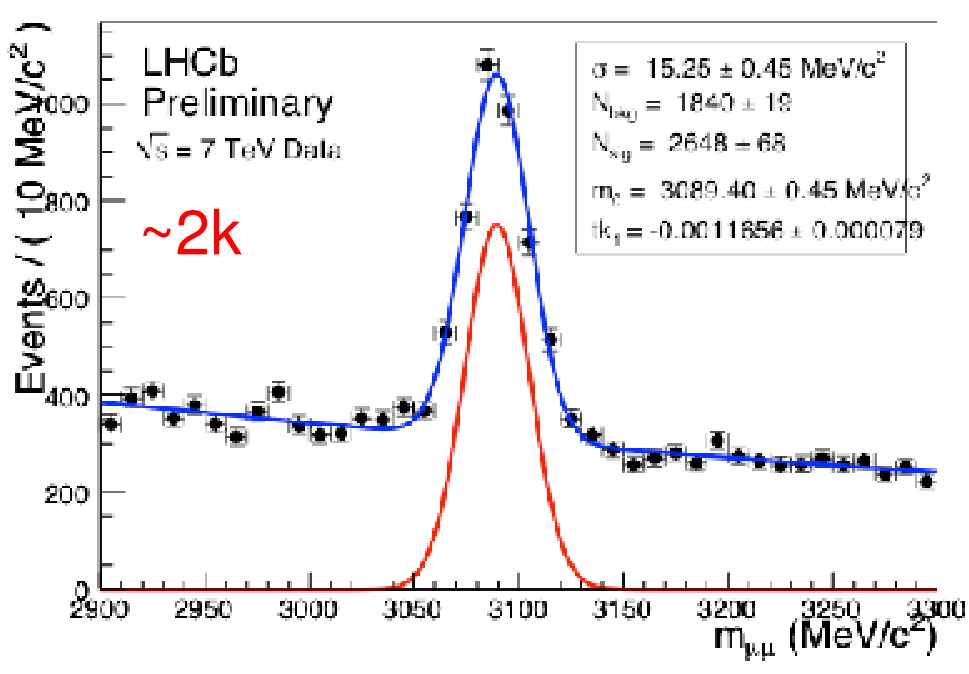

$2 / 07 / 2010$

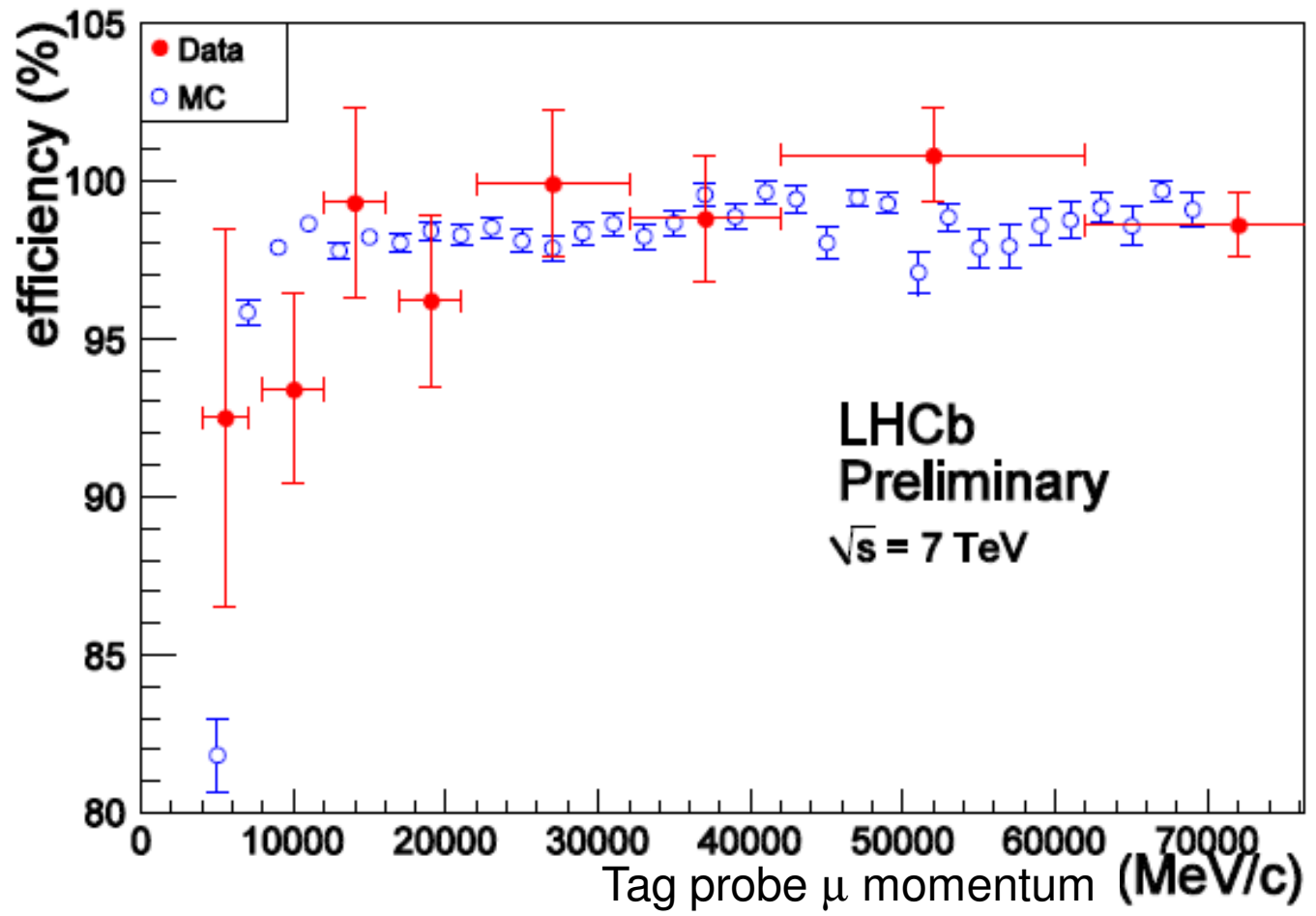

Jeremy Dickens 


\section{$\mathrm{J} / \psi \rightarrow \mu \mu:$ Muon ID}

- $\mathrm{J} / \psi$ "tag and probe"

- $\varepsilon(\mu)=(97.3 \pm 1.2) \%$

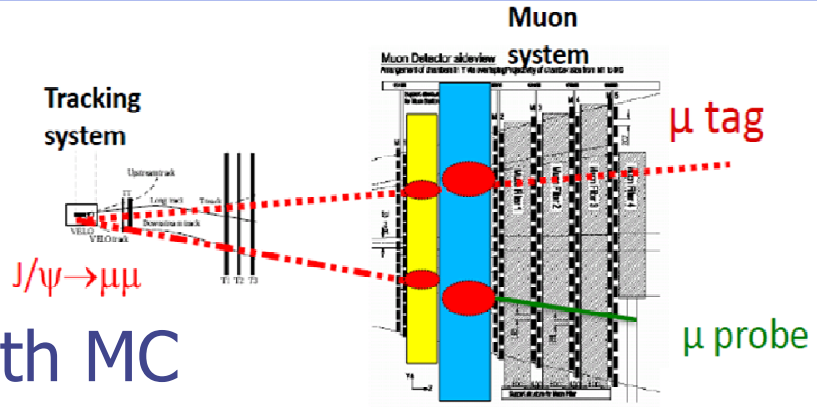

- $\pi \rightarrow \mu$ mis-id (from $K_{S}$ ) in good agreement with MC

- $p \rightarrow \mu$ mis-id (from $\Lambda$ ) in good agreement with MC
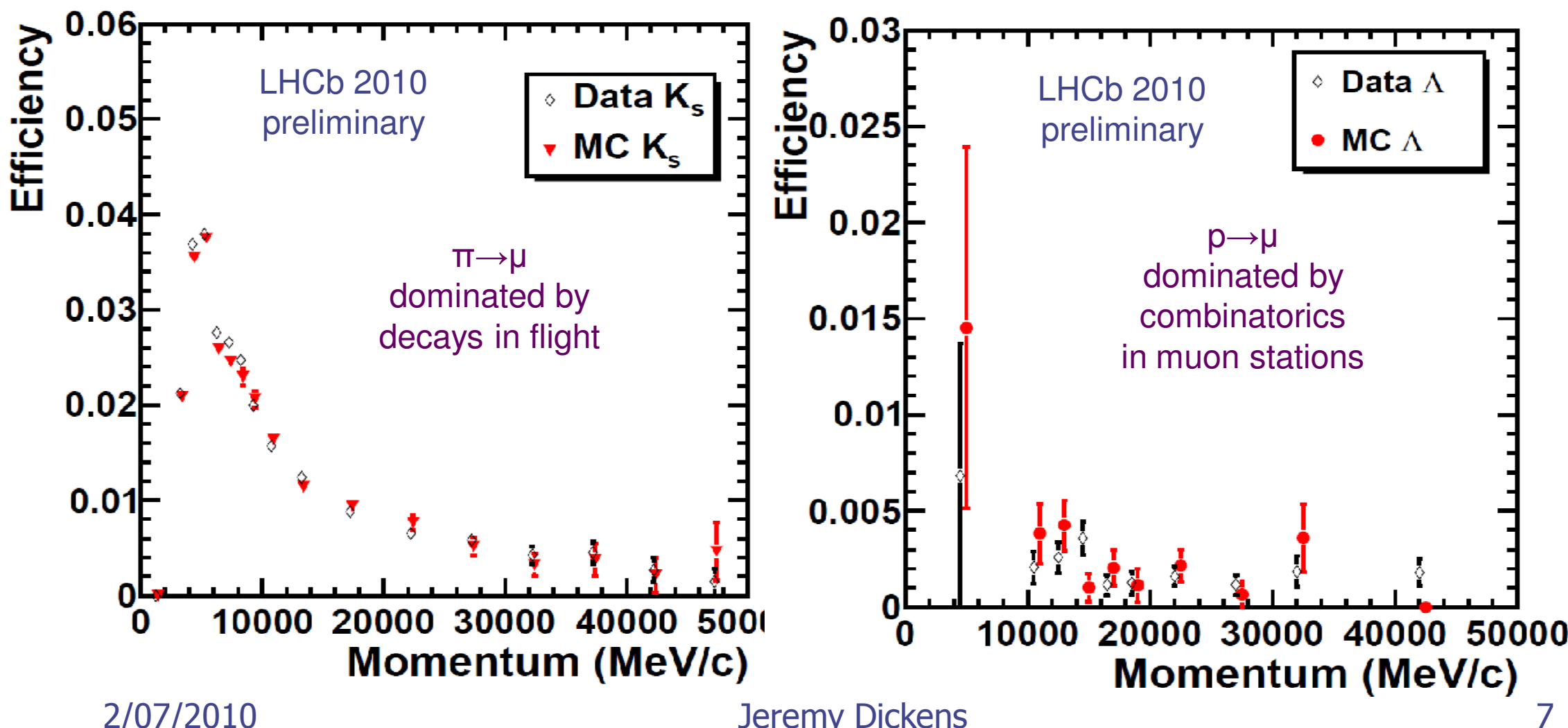


\section{$\mathrm{J} / \psi \rightarrow \mu \mu:$ hints of beauty?}

- Tail in $\mathrm{J} / \psi$ (pseudo) proper time $\Rightarrow \mathrm{B}$ candidates?

Signal window \& normalized sideband
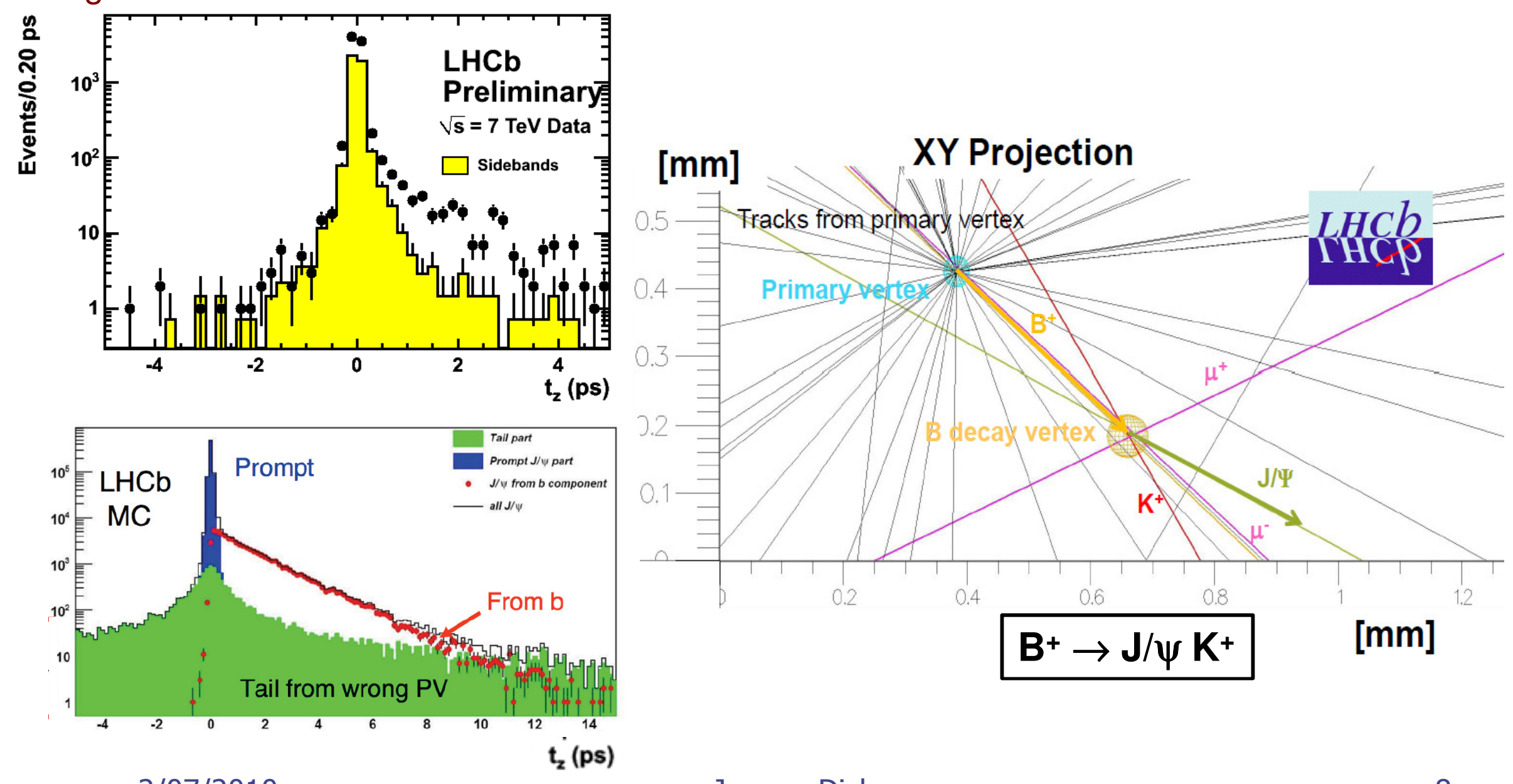

$2 / 07 / 2010$ 


\section{Beauty}

- Handful of B candidates observed

- Hints in $\mathrm{J} / \psi$ channels (expect less than $~ 10$ candidates)

- Fully reconstructed candidates

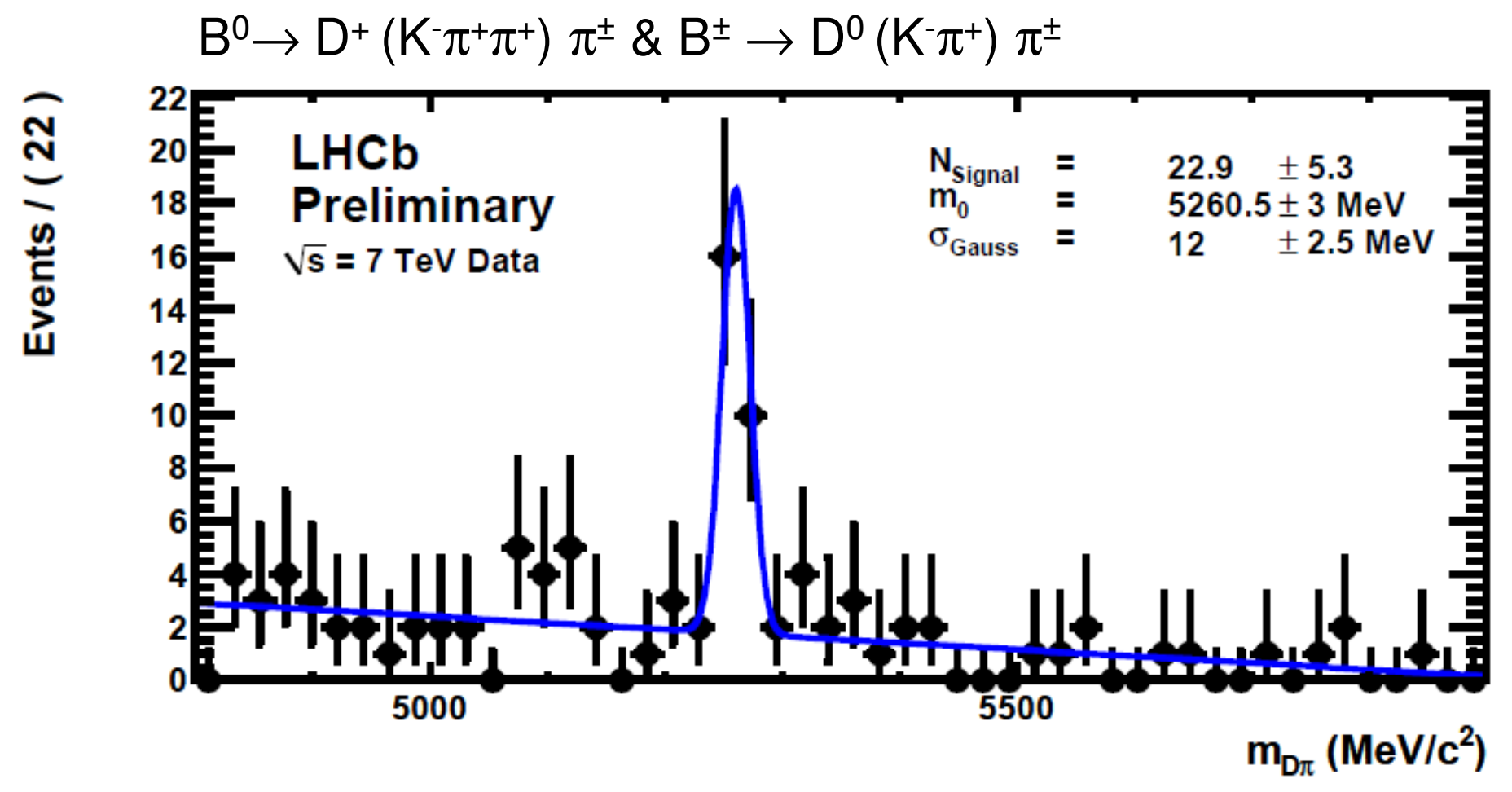




\section{$\mathrm{B}_{\mathrm{s}} \rightarrow \mu \mu$}

- Very rare SM branching ratio: (3.35 \pm 0.32$) \times 10^{-9}$ nep-ph/0604057

- Sensitive to NP - could be enhanced by up to factor 100 in SUSY

- An improved limit / observation should constrain potential NP
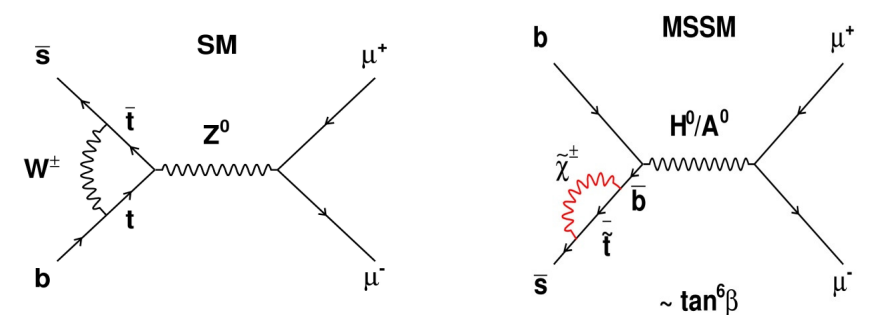

- LHCb: 3D binned likelihood analysis

- Invariant mass, PID, geometric likelihoods

- Uncorrelated variables
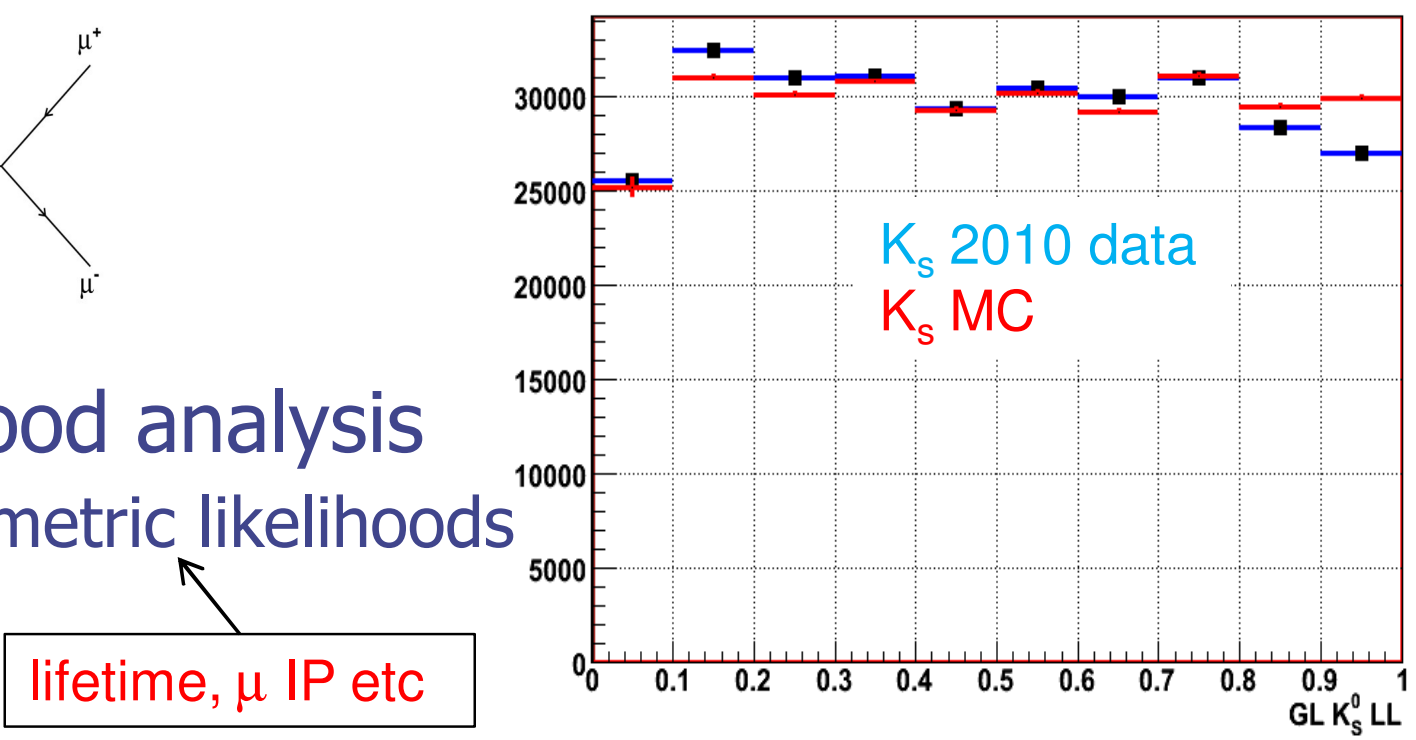

- Calibrated from control channels - little MC input

- Loose selection to remove clear sources of background

- Muon ID is crucial to suppress background from B $\rightarrow$ hh

- Remainder dominated by combinations semi-leptonic B-decays

- Measure branching ratio with respect to $\mathrm{B} \rightarrow \mathrm{J} / \psi \mathrm{K}$ and $\mathrm{B} \rightarrow \mathrm{K} \pi$ 


\section{$\mathrm{B}_{\mathrm{s}} \rightarrow \mu \mu$}

- Current studies suggest MC sensitivity is realistic

- $200 \mathrm{pb}^{-1}$ (2010): improve on expected Tevatron limits $\left(8 \mathrm{fb}^{-1}\right)$

- $1 \mathrm{fb}^{-1}$ (2011): exclusion down to $\sim 7 \times 10^{-9}$

- $10 \mathrm{fb}^{-1}$ for $5 \sigma \mathrm{SM}$ observation (14TeV)
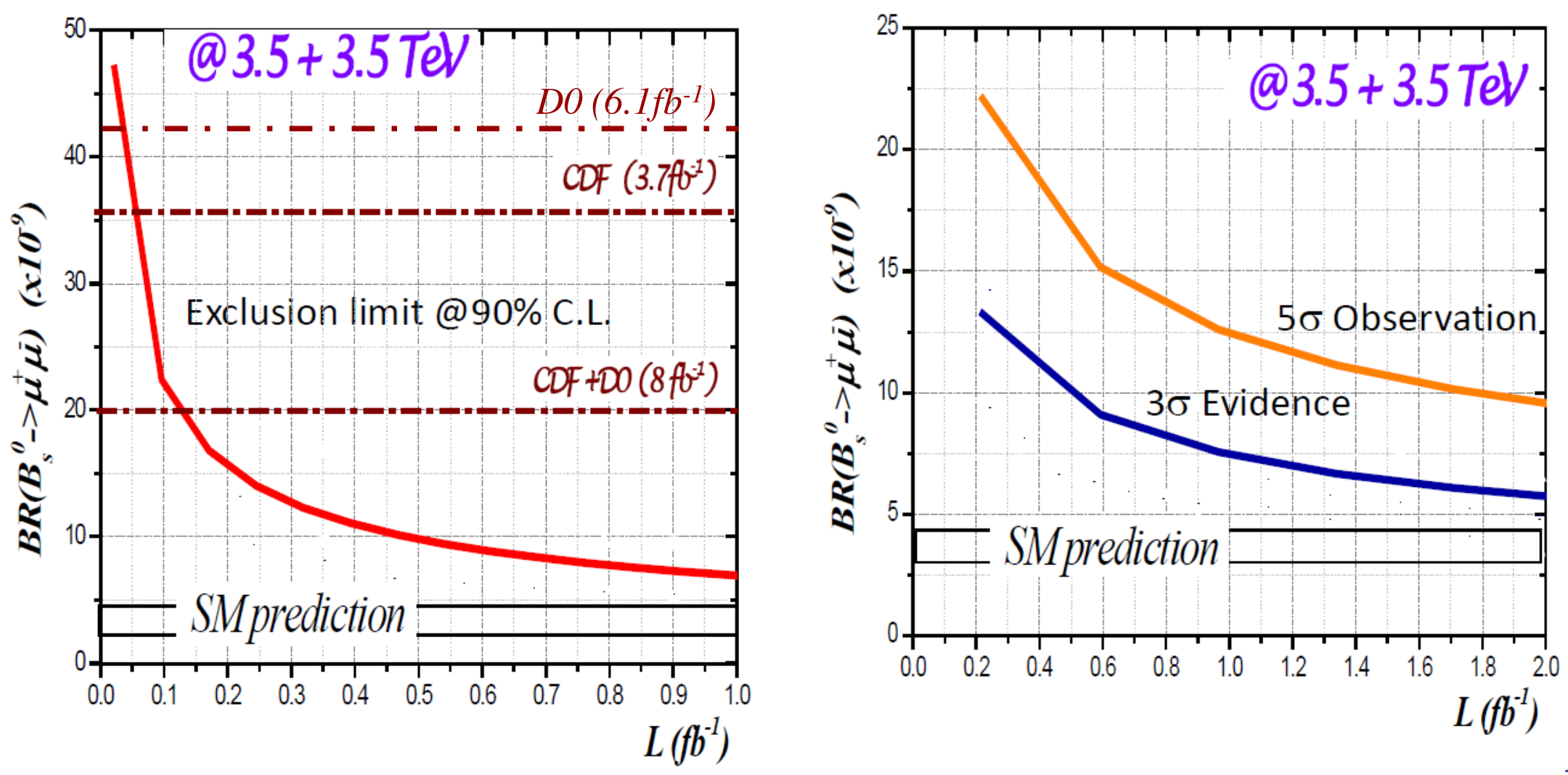


\section{$\mathrm{B}_{\mathrm{d}} \rightarrow \mathrm{K}^{*} \mu \mu$}

- Rare FCNC decay $\rightarrow$ no signal observed at LHCb yet(!)

- PDG branching ratio: $(9.8 \pm 2.1) \times 10^{-7}$

- In broad agreement with SM predictions

- Possibility to discover NP in angular distributions

- Many angular observables sensitive to NP

- Focus on forward-backward asymmetry $\left(A_{F B}\right)$ as a function of $\mathrm{q}^{2}$ (di-muon mass squared)
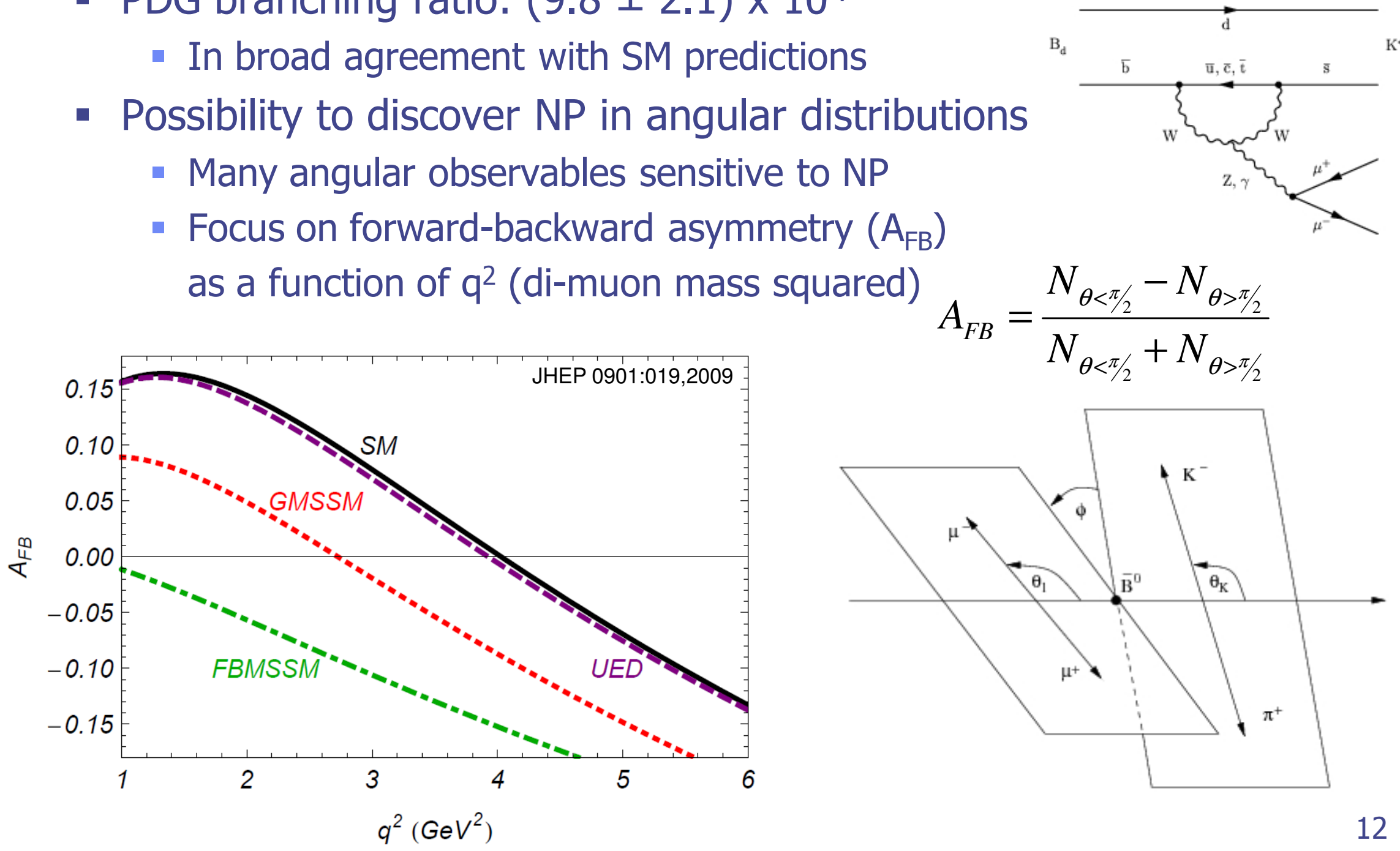

$A_{F B}=\frac{N_{\theta<\pi / 2}-N_{\theta>\pi / 2}}{N_{\theta<\pi / 2}+N_{\theta>\pi / 2}}$

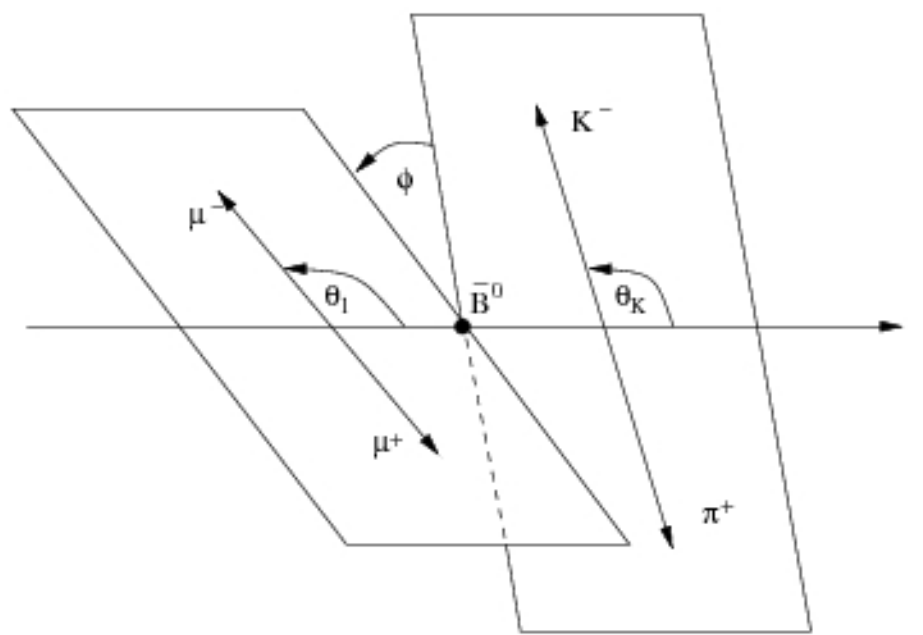




\section{$B_{d} \rightarrow K^{*} \mu \mu: A_{F B}$}

- Full angular fit $\left(\theta_{\mathrm{L}}, \theta_{\mathrm{K}}\right.$ and $\left.\phi\right)$ requires $>2 \mathrm{fb}^{-1}$

- Projection fits to $\theta_{\mathrm{L}}, \theta_{\mathrm{K}} \rightarrow$ still measure $A_{\mathrm{FB}}\left(F_{\mathrm{L}}\right)$

- LHCb statistical precision (assume Belle central value)

\begin{tabular}{|c|c|}
\hline $\begin{array}{c}\text { Data } \\
\left(\mathrm{fib}^{-1}\right)\end{array}$ & $\begin{array}{c}\text { SM } \\
\text { exclusion }\end{array}$ \\
\hline 0.1 & $1.5 \sigma$ \\
\hline 0.3 & $2.4 \sigma$ \\
\hline 0.5 & $3.1 \sigma$ \\
\hline
\end{tabular}

Green band - SM curve

Purple band - SM average

Red points - BaBar

Blue points - Belle

Black points - $\mathrm{LHCb}(\mathrm{MC})$

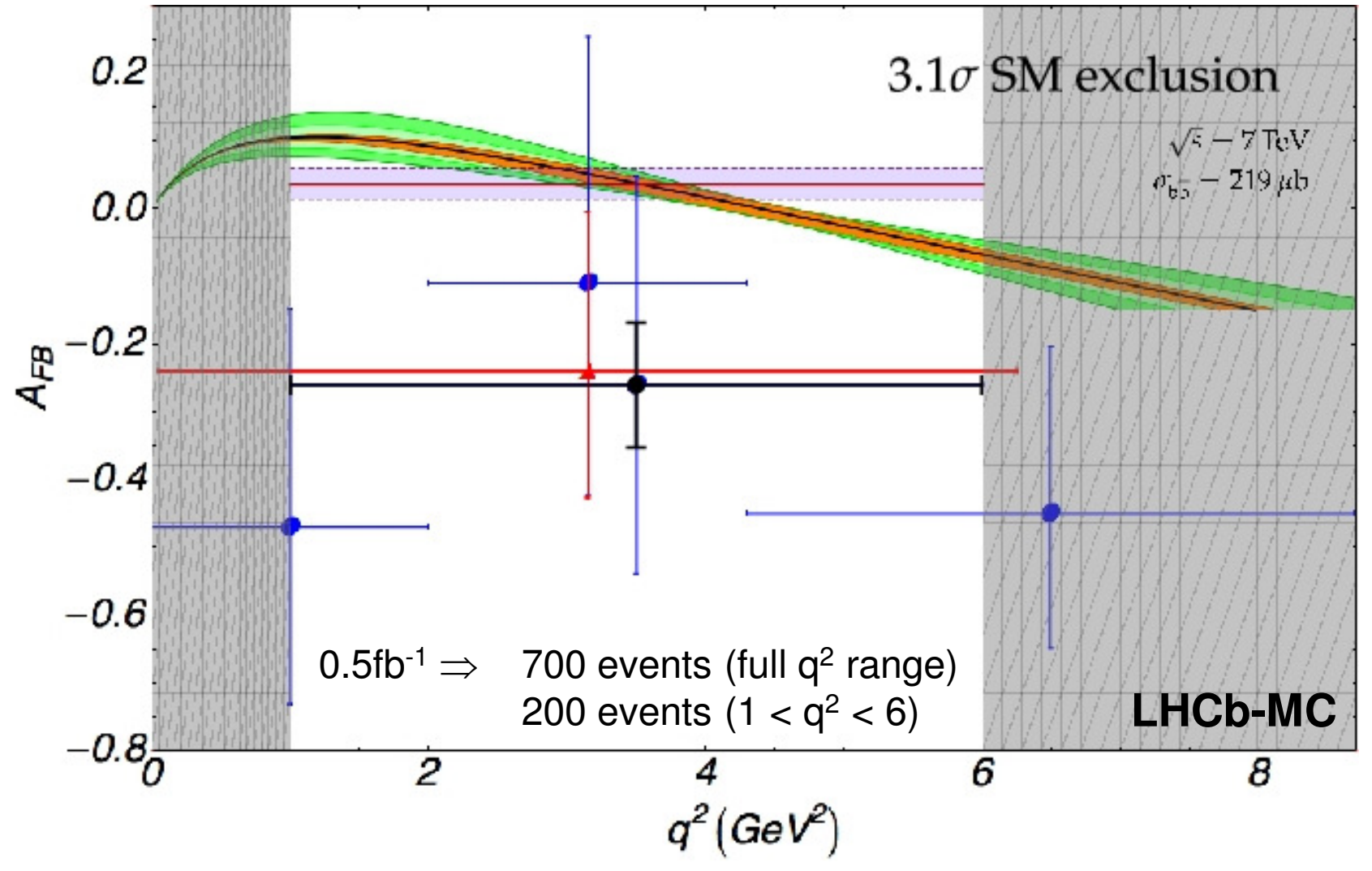




\section{$\mathrm{B}_{\mathrm{s}} \rightarrow \mathrm{J} / \psi \phi$}

- Measure mixing-induced CP-violation in $b \rightarrow$ ccs decay

- Mixing phase $\phi_{\mathrm{s}}=\phi_{\mathrm{s}}^{\mathrm{SM}}+\phi_{\mathrm{s}}^{\mathrm{NP}}$

- $\phi_{\mathrm{S}}{ }^{\mathrm{SM}}=-2 \beta_{\mathrm{S}}=0.036 \pm 0.002$ (CKM fitter)

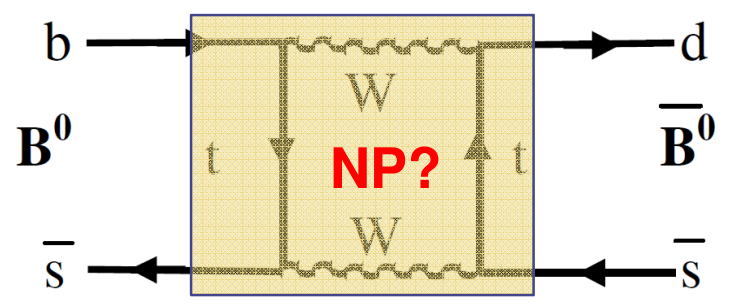

- Measurement requires

- Flavour tagging

- Time dependent analysis

- Angular analysis

- Good prospect for NP

- $\sigma\left(\phi_{\mathrm{S}}\right)=0.14\left(200 \mathrm{pb}^{-1}\right)$

- $\sigma\left(\phi_{\mathrm{S}}\right)=0.07\left(1 \mathrm{fb}^{-1}\right)$

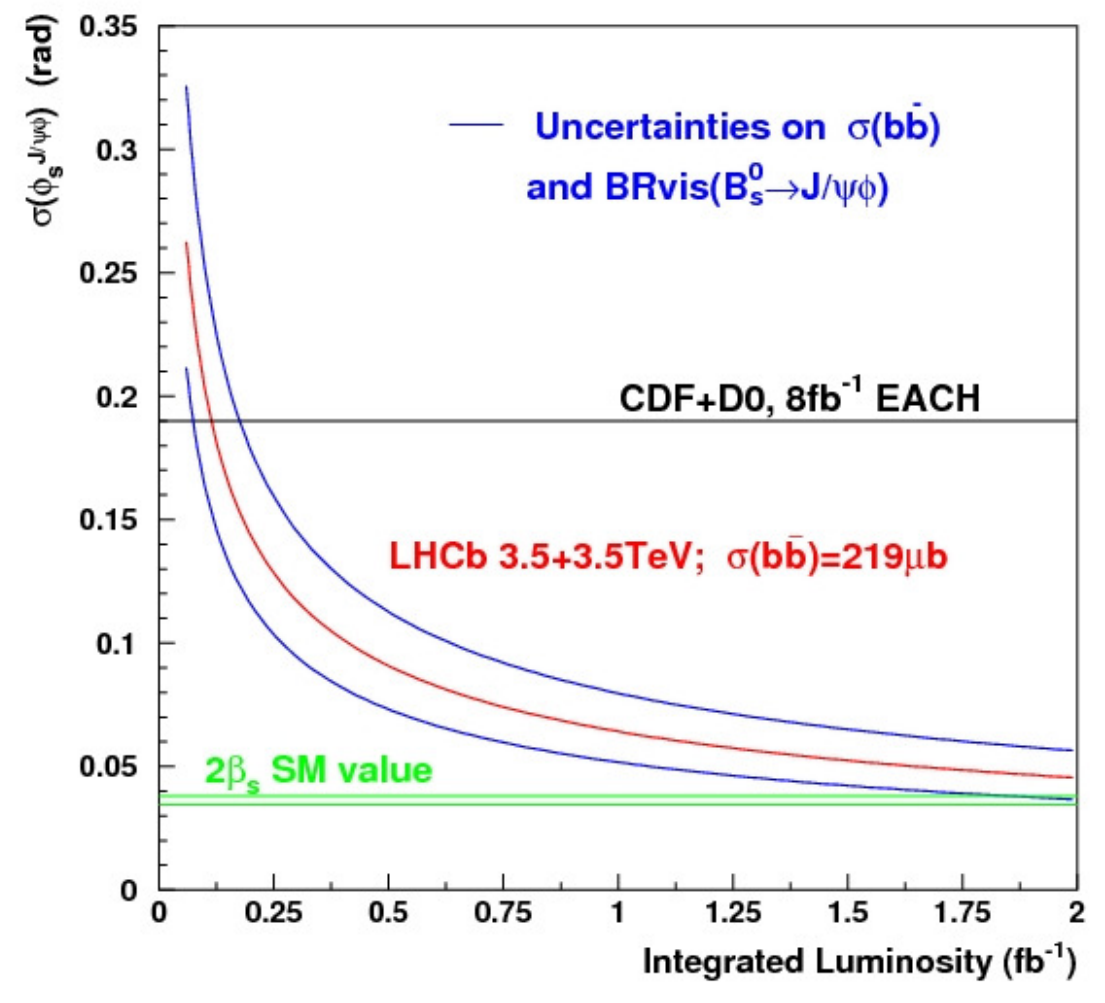




\section{Conclusions}

- LHCb physics program progressing well

- Measurements in charm sector

- Direct CPV

- $\mathrm{y}_{\mathrm{CP}}$

- Observation of B particles

- Good prospects for NP discovery in $B_{s} \rightarrow \mu \mu, B_{d} \rightarrow K^{*} \mu \mu, B_{s} \rightarrow J / \psi \phi$

- Await more data! 


\section{Backup}




\section{Strangeness}

- Large sample of strange particles

- $\mathrm{K}_{\mathrm{S}}$ cross section, $\Lambda$ /anti- $\Lambda$ ratio etc (see talk by $\mathrm{F}$. Dettori)
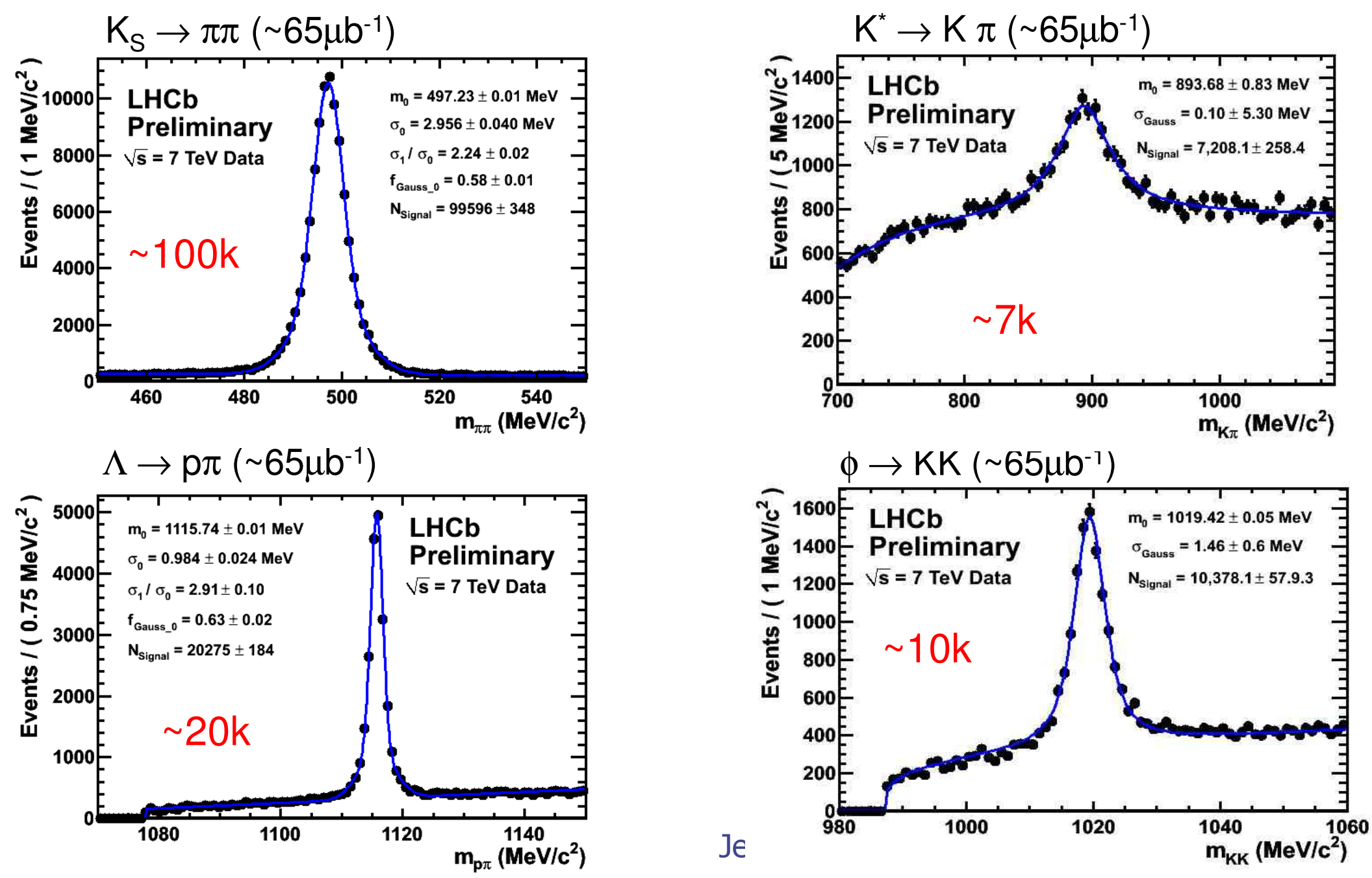


\section{RICH PID performance}

- $\phi$ is useful to measure the kaon PID performance

- 2009 data (450 GeV) - add PID information $\rightarrow$ see a $\phi$ peak
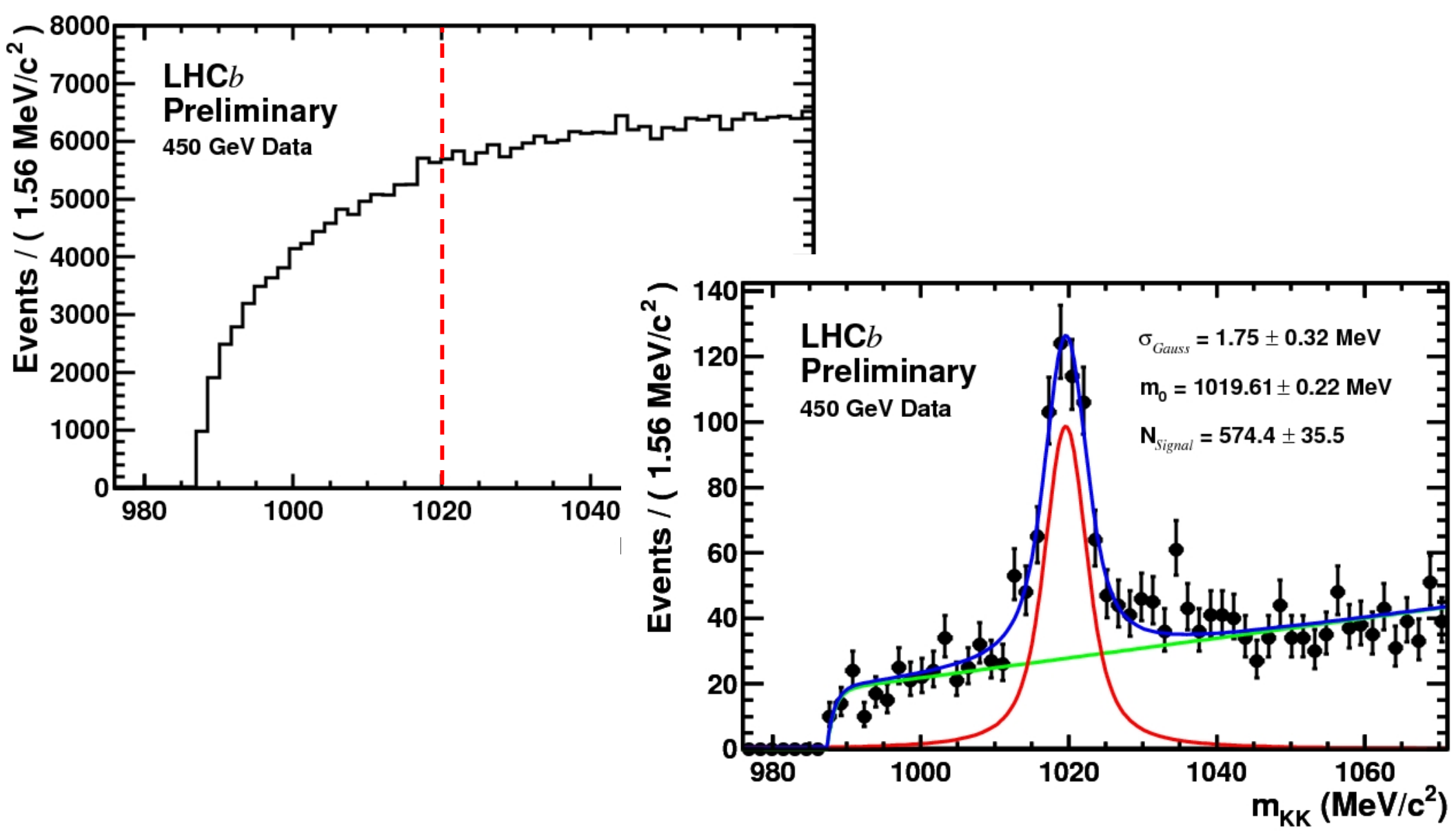

Jeremy Dickens 


\section{RICH PID performance}

- $\phi$ is useful to measure the kaon PID performance

- 2009 data (450 GeV) - add PID information $\rightarrow$ see a $\phi$ peak

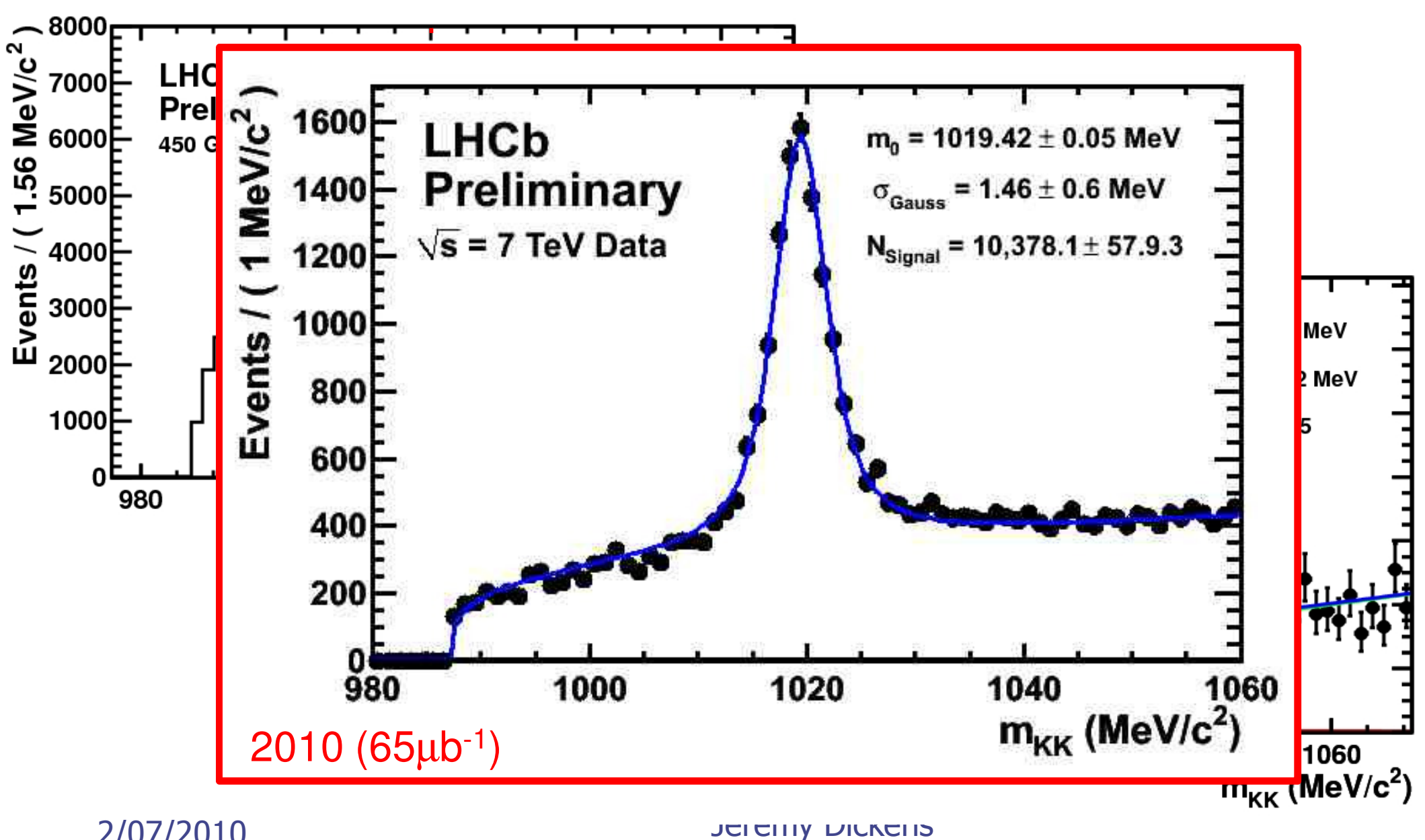




\section{RICH PID performance}

- $\phi$ is useful to measure the kaon PID performance

- 2010 data (7 TeV) - "tag and probe" to extract PID performance

$$
\Delta \log \mathcal{L}(\mathrm{K}-\pi)>0
$$

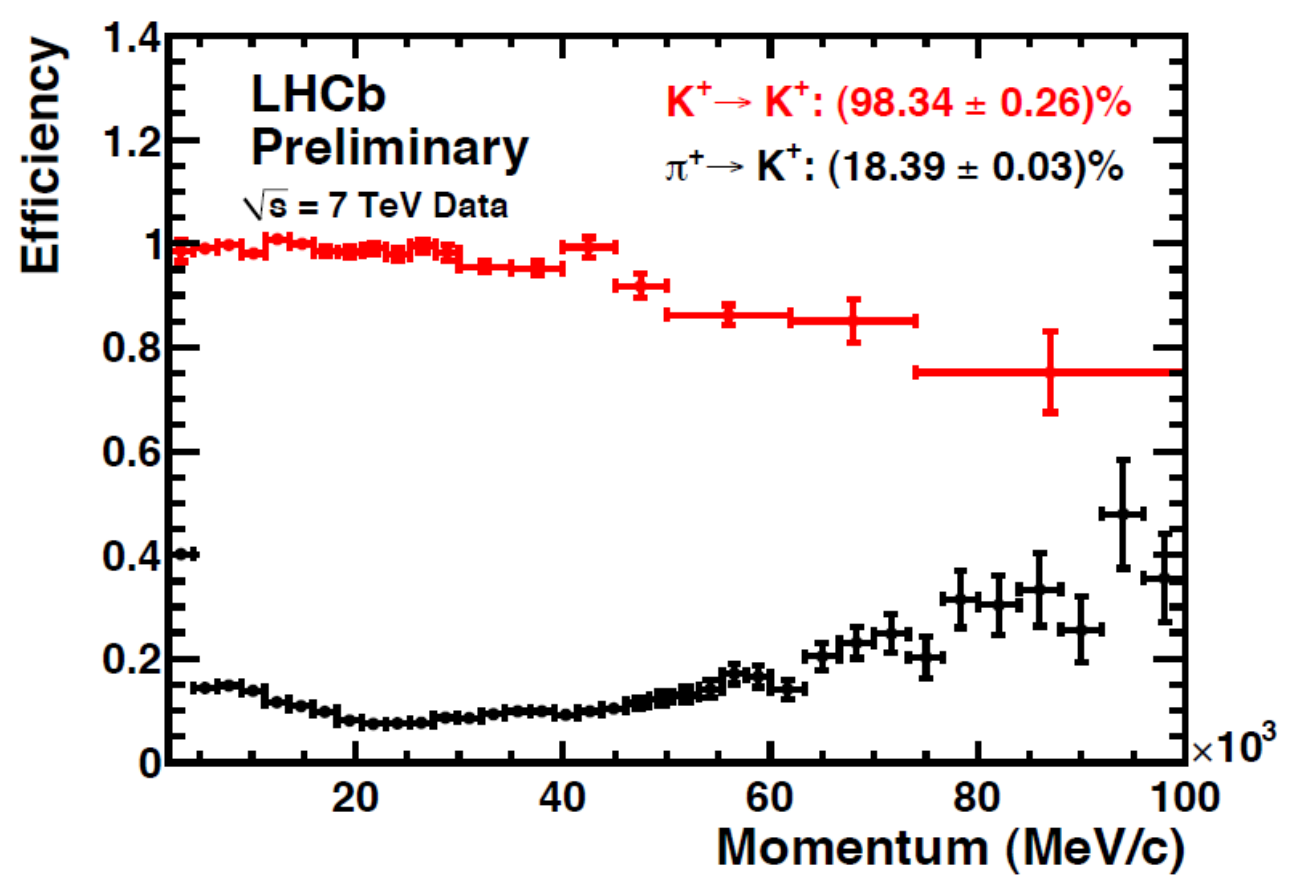

- Work still ongoing with alignment / calibration

- Cherenkov angle resolutions close to expectations 


\section{LHCb Trigger}

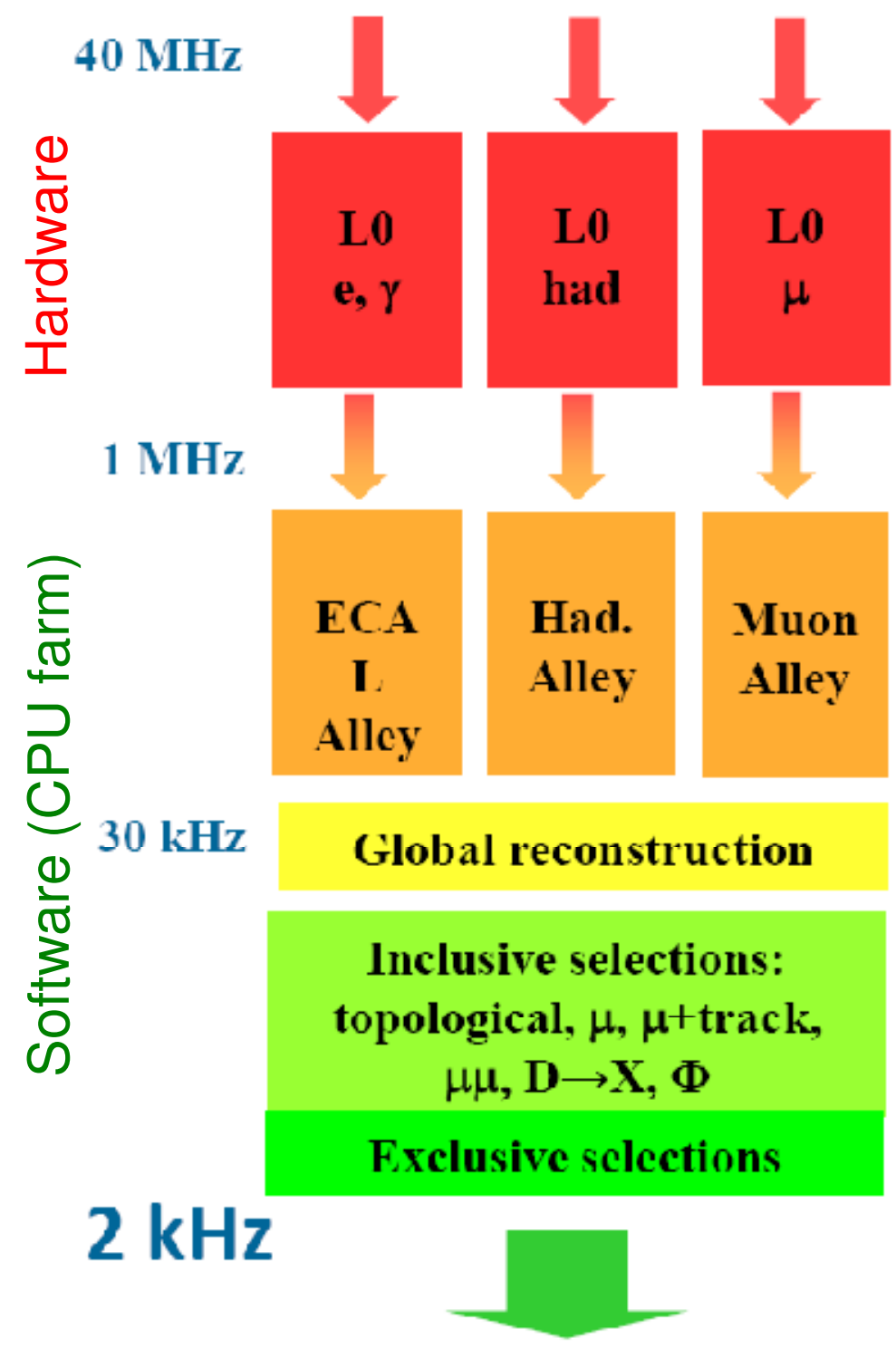




\section{LHCb Trigger}

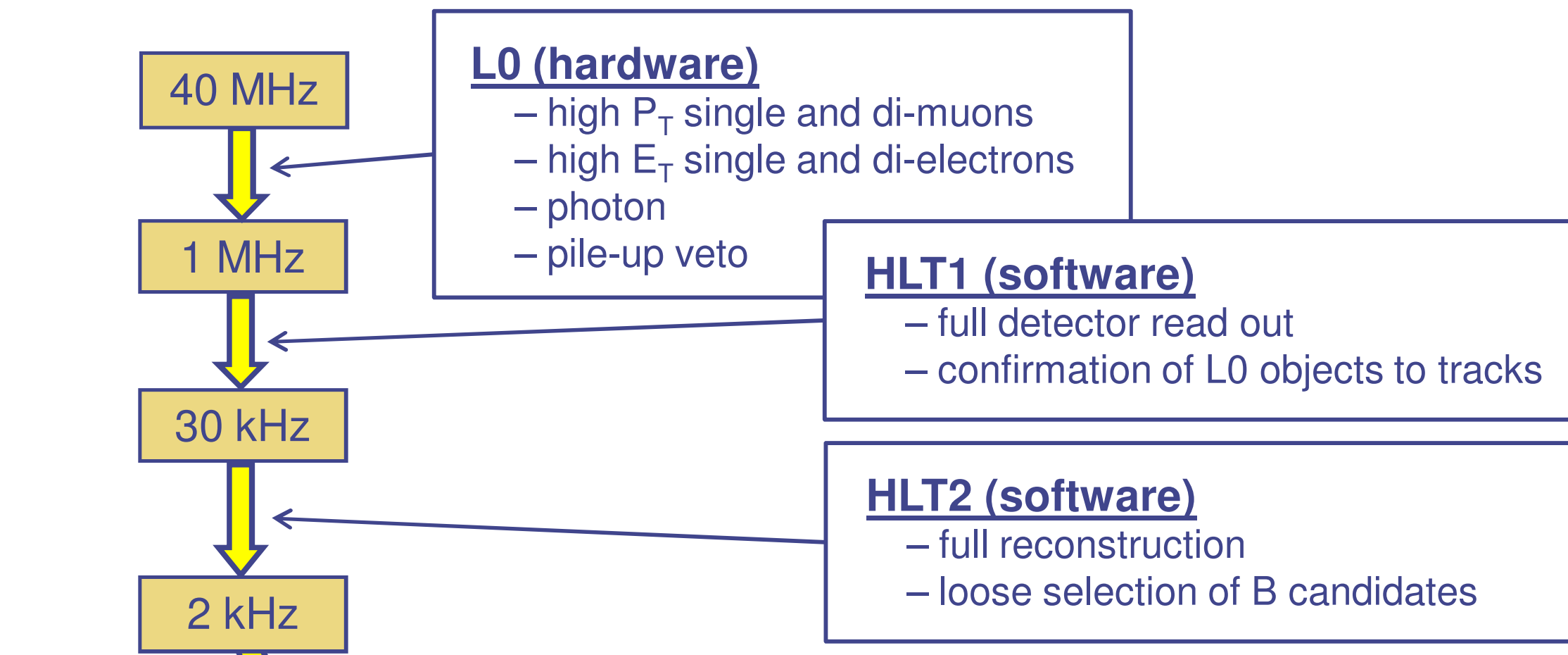

\section{Exclusive selections,}

Inclusive $(\mu, \mu+$ track, $\mu \mu$, topological $\mathrm{D} \rightarrow \mathrm{X}, \phi$ ) 


\section{LHCb Trigger}

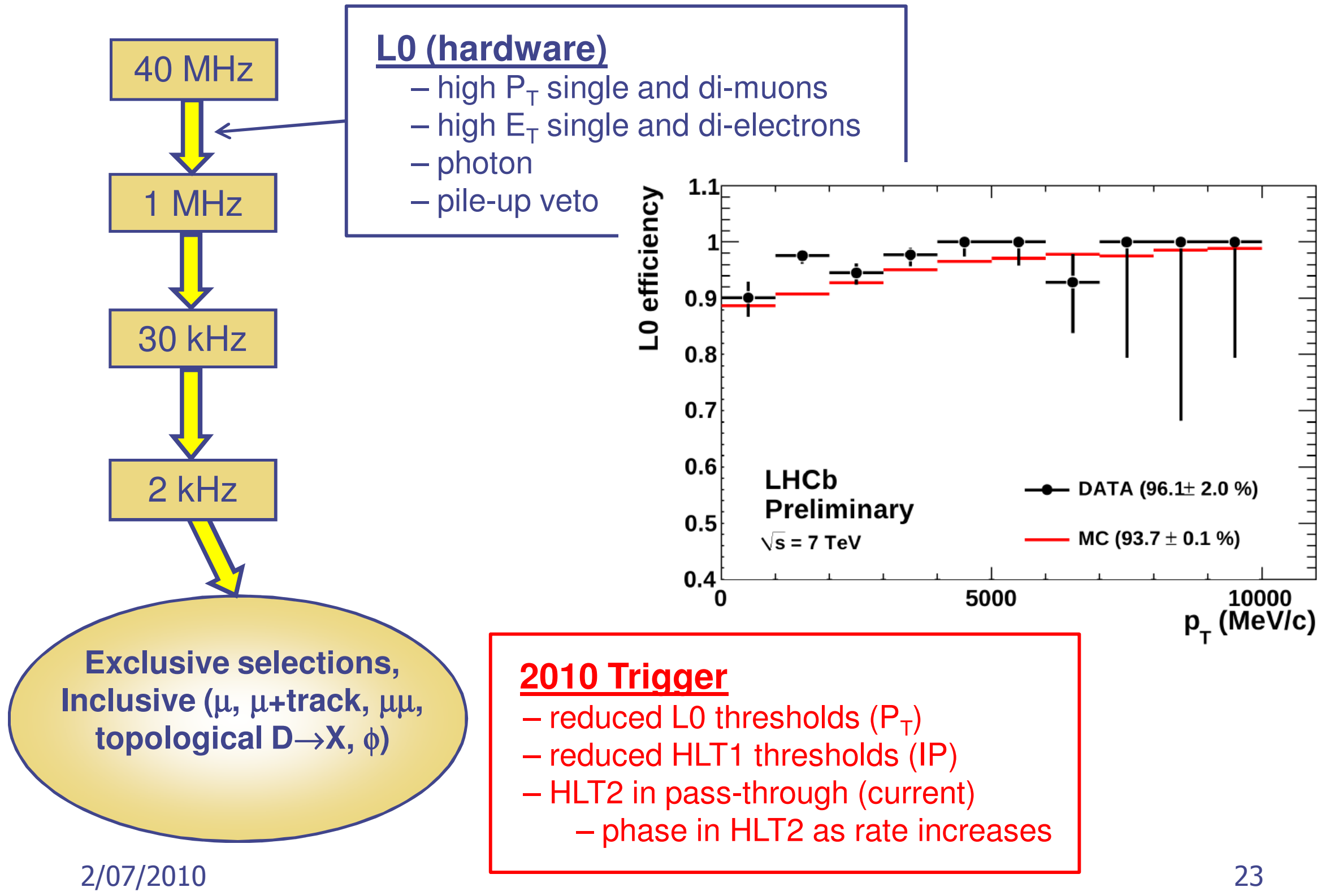




\section{Cuts for plots}

- $\mathrm{K}_{\mathrm{S}} \rightarrow \pi \pi$

- $\Lambda \rightarrow \mathrm{p} \pi$ veto (8 MeV)

- Vertex $\chi^{2} /$ dof $<15$

- Vertex-z < $200 \mathrm{~cm}$

- $v>3.0$

- $\Lambda \rightarrow p \pi \downarrow \log _{10}\left(\frac{I P_{+} I P_{-}}{I P_{V 0}}\right)$

- $v>2.0$

- Vertex $\chi^{2} /$ dof $<15$

- Vertex-z < $200 \mathrm{~cm}$

- $\mathrm{K}_{\mathrm{s}} \rightarrow \pi \pi$ veto (17.5 MeV)
- $\mathrm{K}^{*}(892) \rightarrow \mathrm{K} \pi$

- $\operatorname{IP} \chi^{2}\left(K^{*}\right)<10$

- $\mathrm{FD} \chi^{2}\left(\mathrm{~K}^{*}\right)<20$

- $\mathrm{p}_{\mathrm{T}}\left(\mathrm{K}^{*}\right)>400 \mathrm{MeV}$

- $\operatorname{IP} \chi^{2}(K, \pi)<4$

- Kaon $\Delta_{\mathrm{LL}}(\mathrm{K}-\pi)>30$

- Pion $\Delta_{\mathrm{LL}}(\mathrm{K}-\pi)<-30$

- $\phi \rightarrow \mathrm{KK}$

- Vertex $\chi^{2} /$ dof $<25$

- $\mathrm{FD}^{2}(\phi)<15$

- Kaon $\Delta_{\mathrm{LL}}(\mathrm{K}-\pi)>30$ 


\section{Cuts for plots}

- $\mathrm{J} / \psi \rightarrow \mu \mu$ efficiency

- Tag $\mu: \operatorname{ID}(\mu)$

$$
\begin{aligned}
& \mathrm{p}_{\mathrm{T}}>1.5 \mathrm{GeV} \\
& \mathrm{p}>6 \mathrm{GeV}
\end{aligned}
$$

- Probe $\mu$ :

\section{Calo(mips)}

1 hit in M2 FoI 


\section{$\mathrm{CPV}$ in $\mathrm{D}+\rightarrow \mathrm{K}_{\mathrm{S}} \pi$}

Cabbibo Allowed

$$
D^{+} \rightarrow K_{s} \pi^{+}
$$$$
D_{s}^{+} \rightarrow K_{s} K^{+}
$$

Cabbibo Suppressed
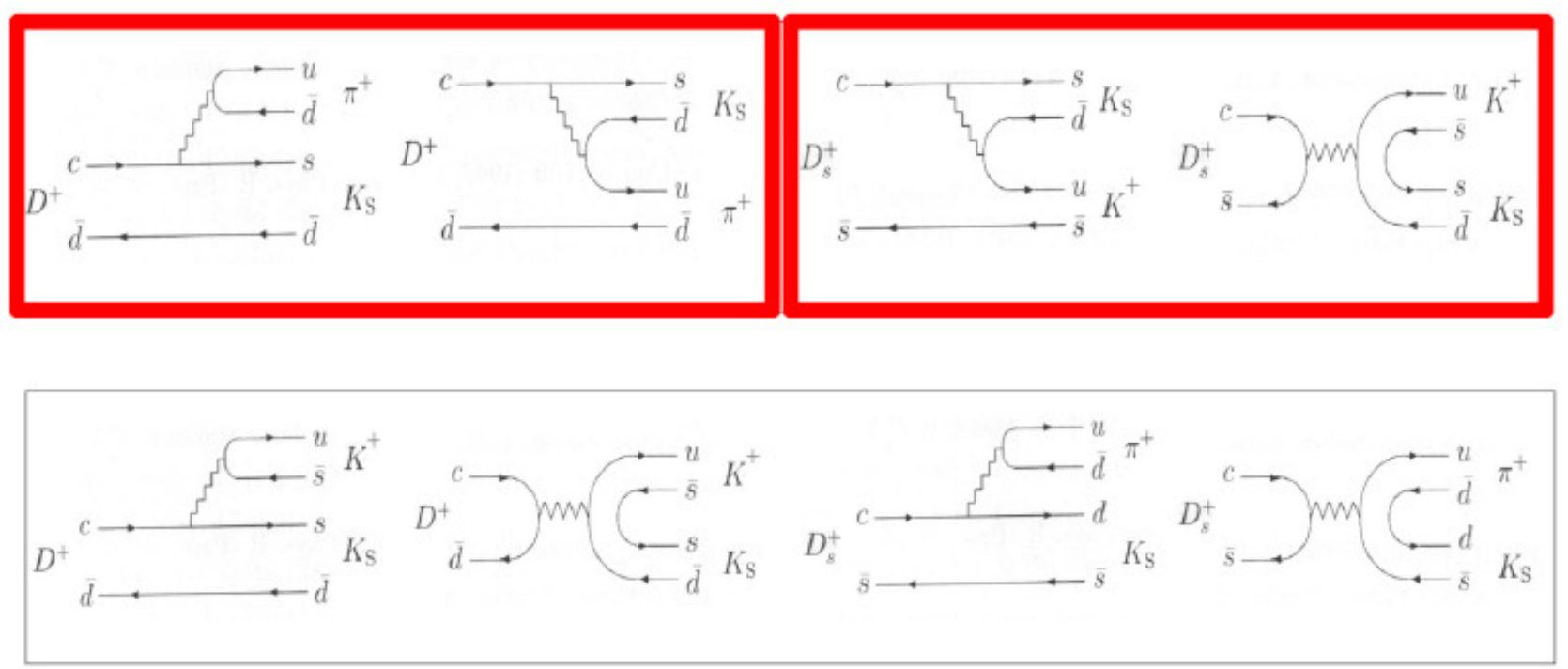

Doubly Cabbibo

Suppressed

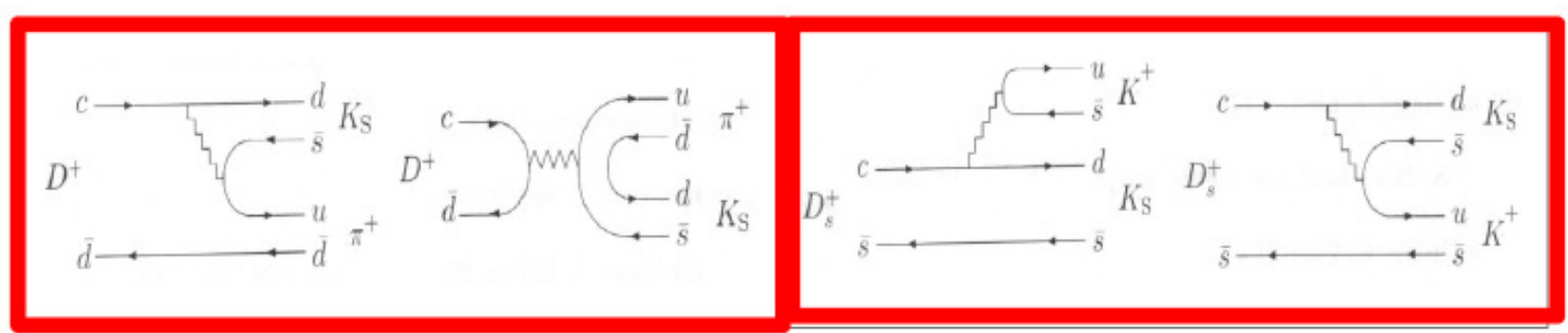




\section{$\mathrm{B}_{\mathrm{s}} \rightarrow \mu \mu$}

- 3 variables:

- Invariant-mass

- Geometric likelihood:

- $B_{s}$ lifetime

- $\mu$ impact parameter significance

- $B_{s}$ impact parameter

- Distance of closest approach of muons

- Isolation

- PID likelihood

- Combination of probabilities as $\mu, \mathrm{K}, \pi$ 


\section{$B_{d} \rightarrow K^{*} \mu \mu$}

- Rare FCNC decay $\rightarrow$ no signal observed at LHCb yet(!)

- Branching ratio (PDG) measured to be $(9.8 \pm 2.1) \times 10^{-6}$

- In broad agreement with SM predictions

- Possibility to discover NP in angular distributions

- Transverse asymmetry $A_{T}{ }^{(2)}$ [Egede, Reece]
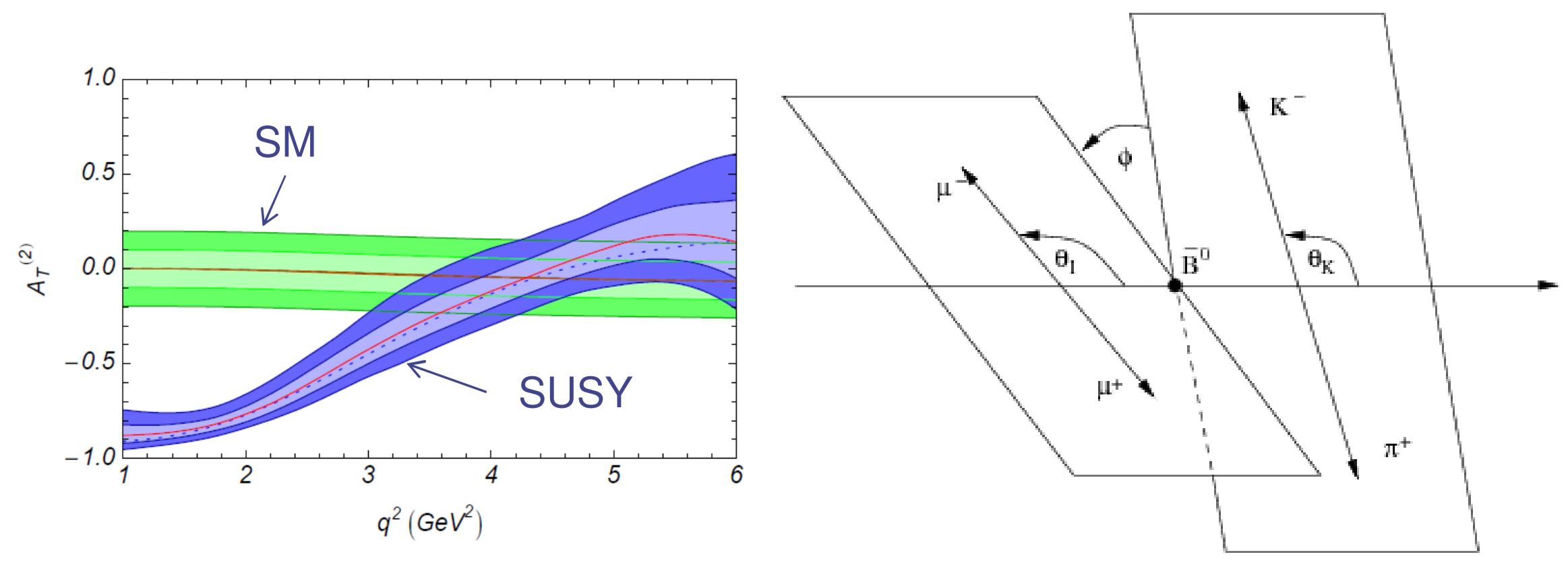


\section{$B_{d} \rightarrow K^{*} \mu \mu$ : Signal Selection}

- Exploit good vertex resolution and PID

- Monte-Carlo Fisher discriminant selection

- $2 \mathrm{fb}^{-1}: \mathrm{S}=6200$ and $\mathrm{B}=1550$, at 7+7 TeV

- 2010/11 run:

Dominated by background from $B$ decays

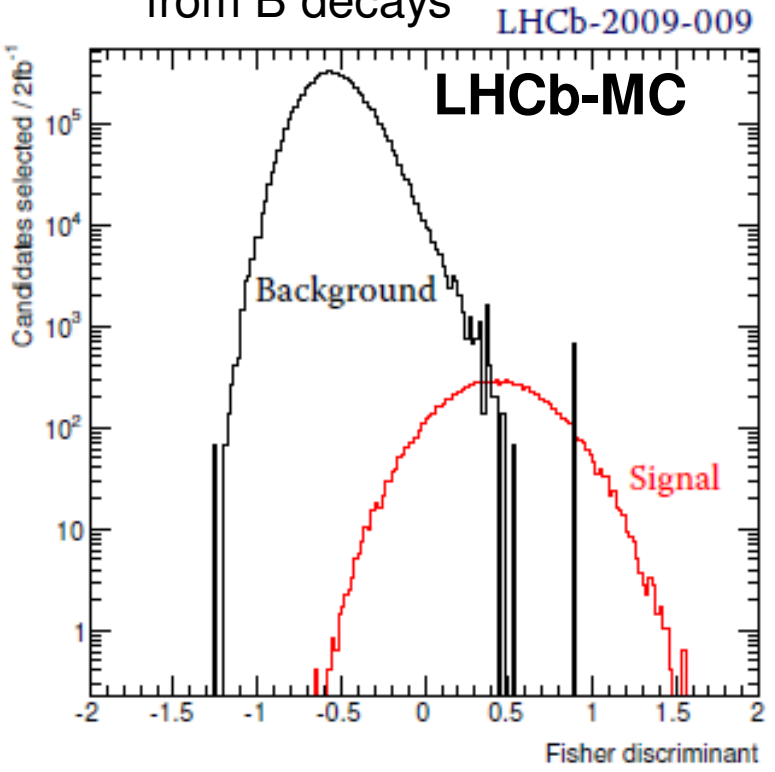

- Hope to collect statistics comparable to other experiments

- Also collect sample of $\sim 64 \mathrm{k} \mathrm{B}_{\mathrm{d}} \rightarrow \mathrm{J} / \psi \mathrm{K}^{*}$ events

- Control channel for $K^{*} \mu \mu$ (eg selection validation / acceptance correction) 


\section{$\mathrm{B}_{\mathrm{d}} \rightarrow \mathrm{K}^{*} \mu \mu:$ Acceptance effects}

- Acceptance varies with $\theta_{\mathrm{L}}$ and $\mathrm{q}^{2} \rightarrow$ bias in $A_{\mathrm{FB}}$

- Sensitive to $\mu^{ \pm} \mathrm{p}_{\mathrm{T}}$ cuts (avoided in selection)

- Dominated by detector geometry

- Requirement of $\mathrm{P}(\mu)>3 \mathrm{GeV}$ to reach muon stations

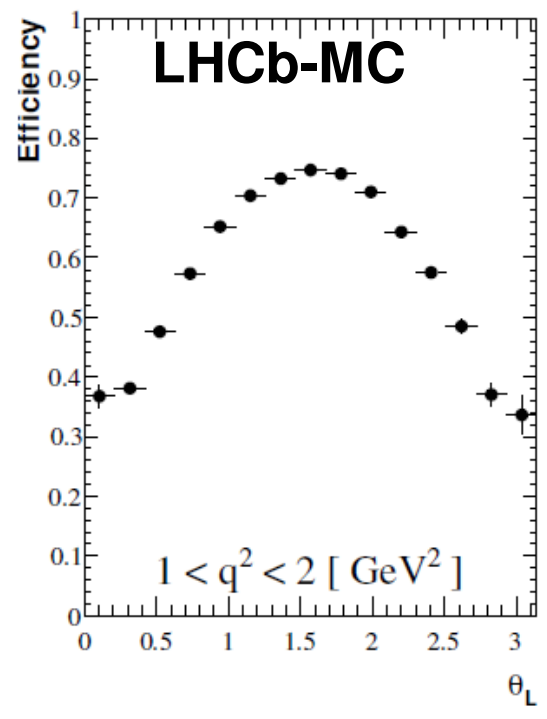

$2 / 07 / 2010$

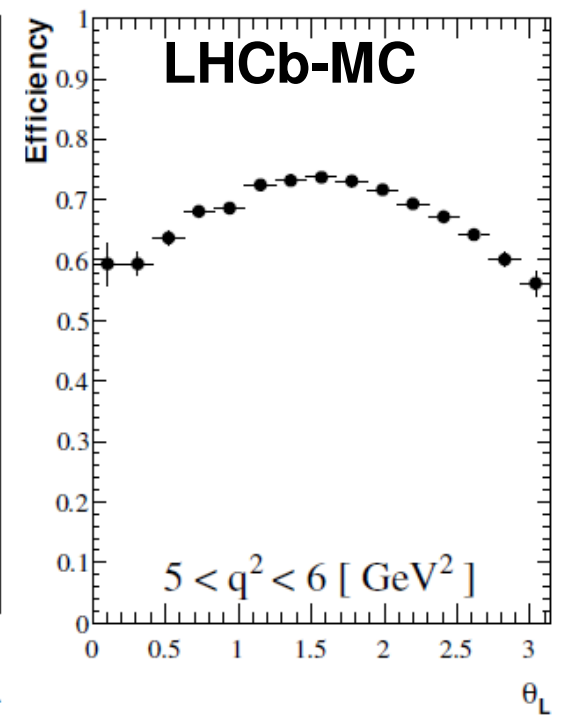

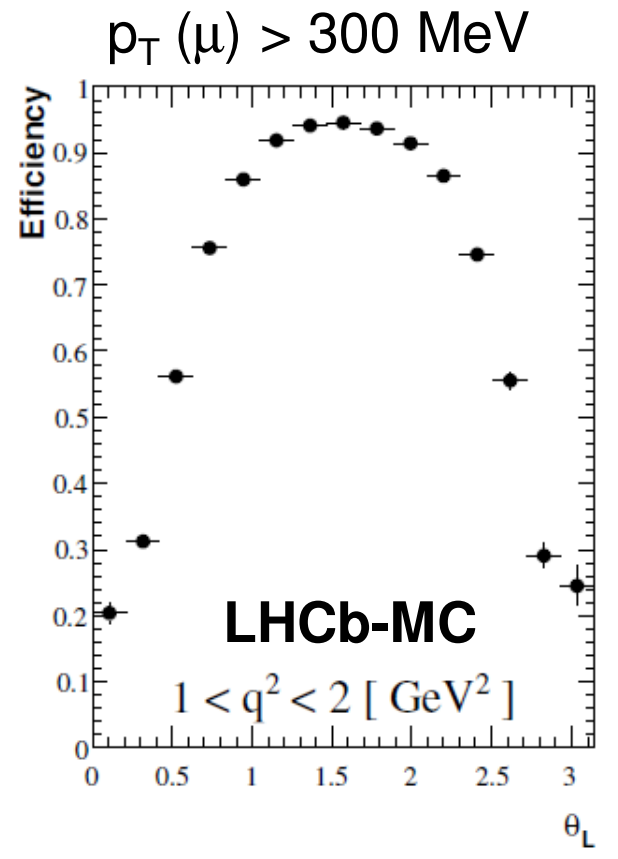

- Shift in $A_{F B}$ expected to be less than statistical precision for 2010/11 run

- Acceptance correction using $\mathrm{J} / \psi \mathrm{K}^{*}$ under investigation 


\section{$B_{d} \rightarrow K^{*} \mu \mu: A_{F B}$}

- Full angular fit $\left(\theta_{\mathrm{L}}, \theta_{\mathrm{K}}\right.$ and $\left.\phi\right)$ requires $>2 \mathrm{fb}^{-1}$

- Just use $\theta_{\mathrm{L}} \rightarrow$ can still measure $A_{\mathrm{FB}}$

- Current results favour flipped $C_{7}(\mathrm{SM})$ : more stats needed BABAR [PRD79:031102,2009]

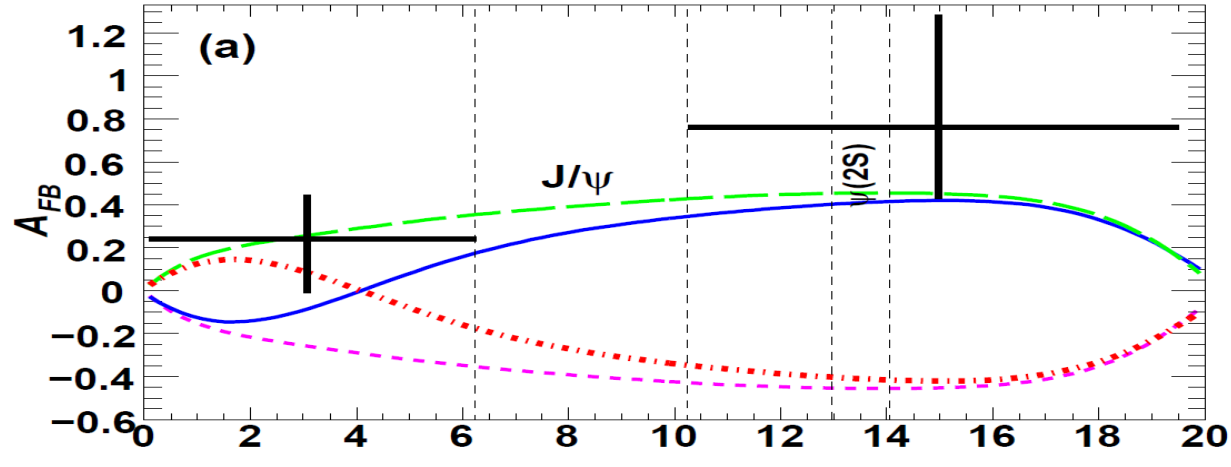

BELLE [PRL103:171801,2009]
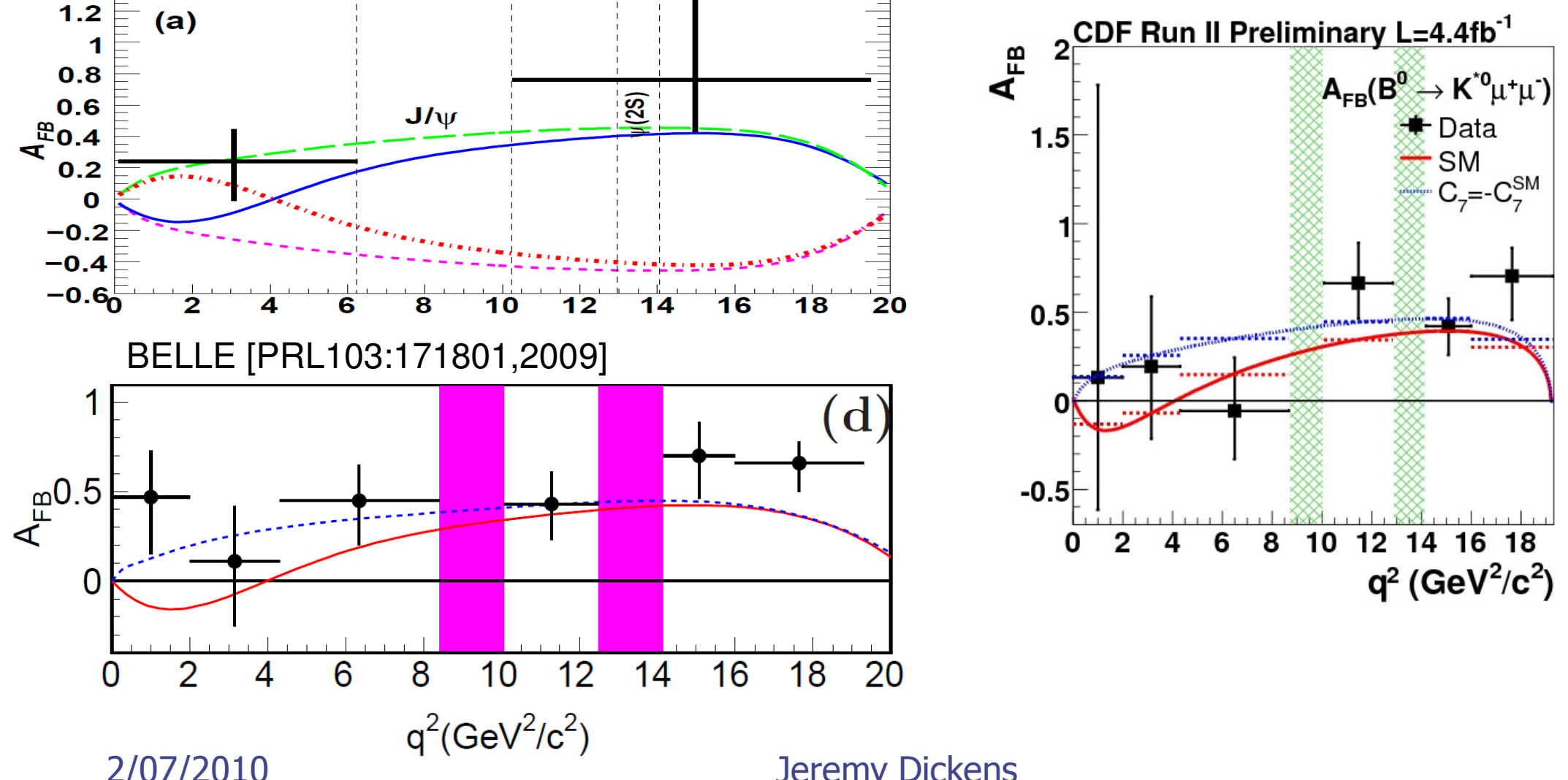

$2 / 07 / 2010$ 


\section{$\mathrm{B}_{\mathrm{d}} \rightarrow \mathrm{K}^{*} \mu \mu: \mathrm{A}_{\mathrm{FB}}$}

- Full angular fit $\left(\theta_{\mathrm{L}}, \theta_{\mathrm{K}}\right.$ and $\left.\phi\right)$ requires $>2 \mathrm{fb}^{-1}$

- Just use $\theta_{\mathrm{L}} \rightarrow$ can still measure $A_{\mathrm{FB}}$

- LHCb statistical precision (assume Belle central value)

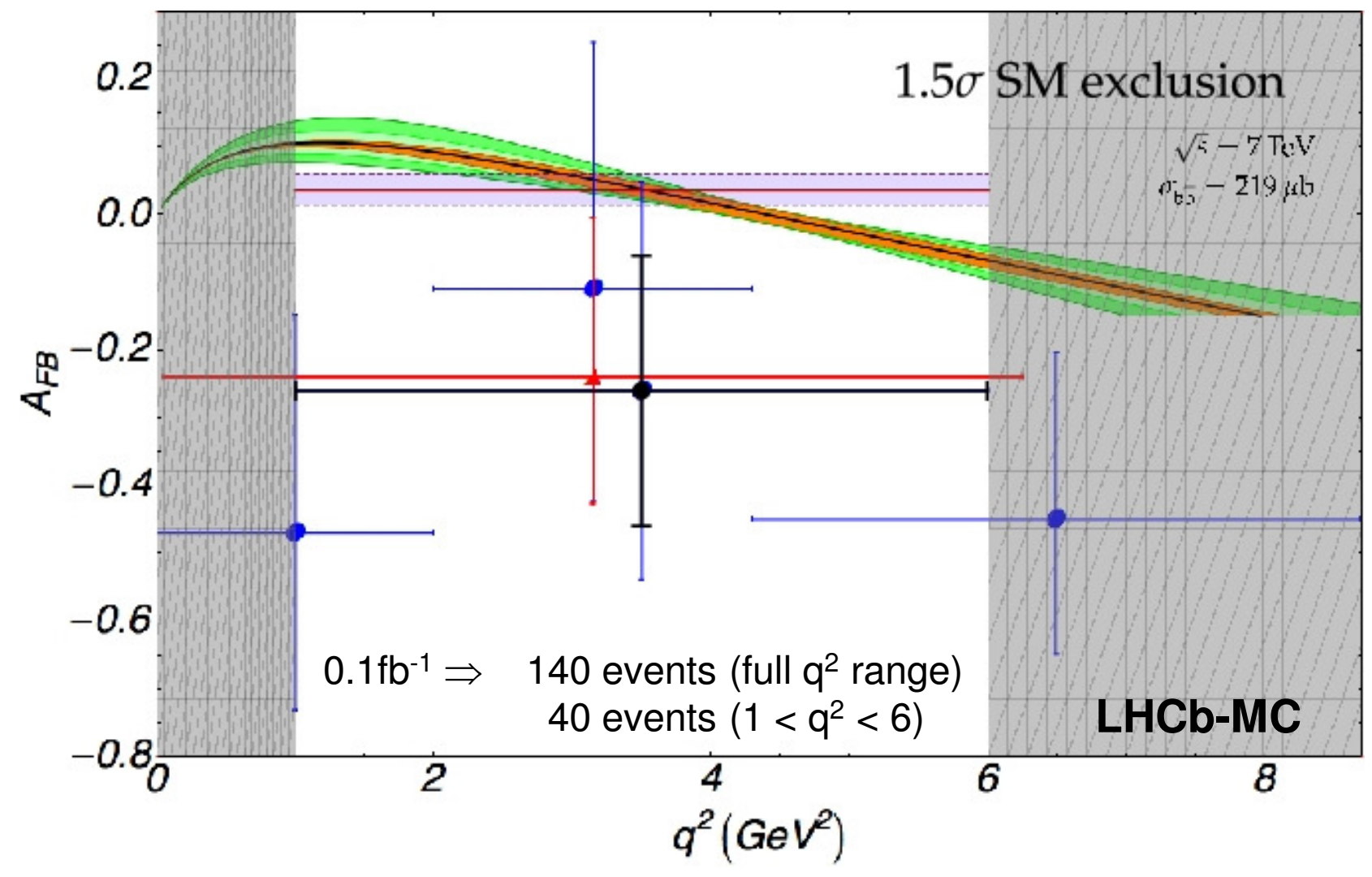




\section{$\mathrm{B}_{\mathrm{d}} \rightarrow \mathrm{K}^{*} \mu \mu: \mathrm{A}_{\mathrm{FB}}$}

- Full angular fit $\left(\theta_{\mathrm{L}}, \theta_{\mathrm{K}}\right.$ and $\left.\phi\right)$ requires $>2 \mathrm{fb}^{-1}$

- Just use $\theta_{\mathrm{L}} \rightarrow$ can still measure $A_{\mathrm{FB}}$

- LHCb statistical precision (assume Belle central value)

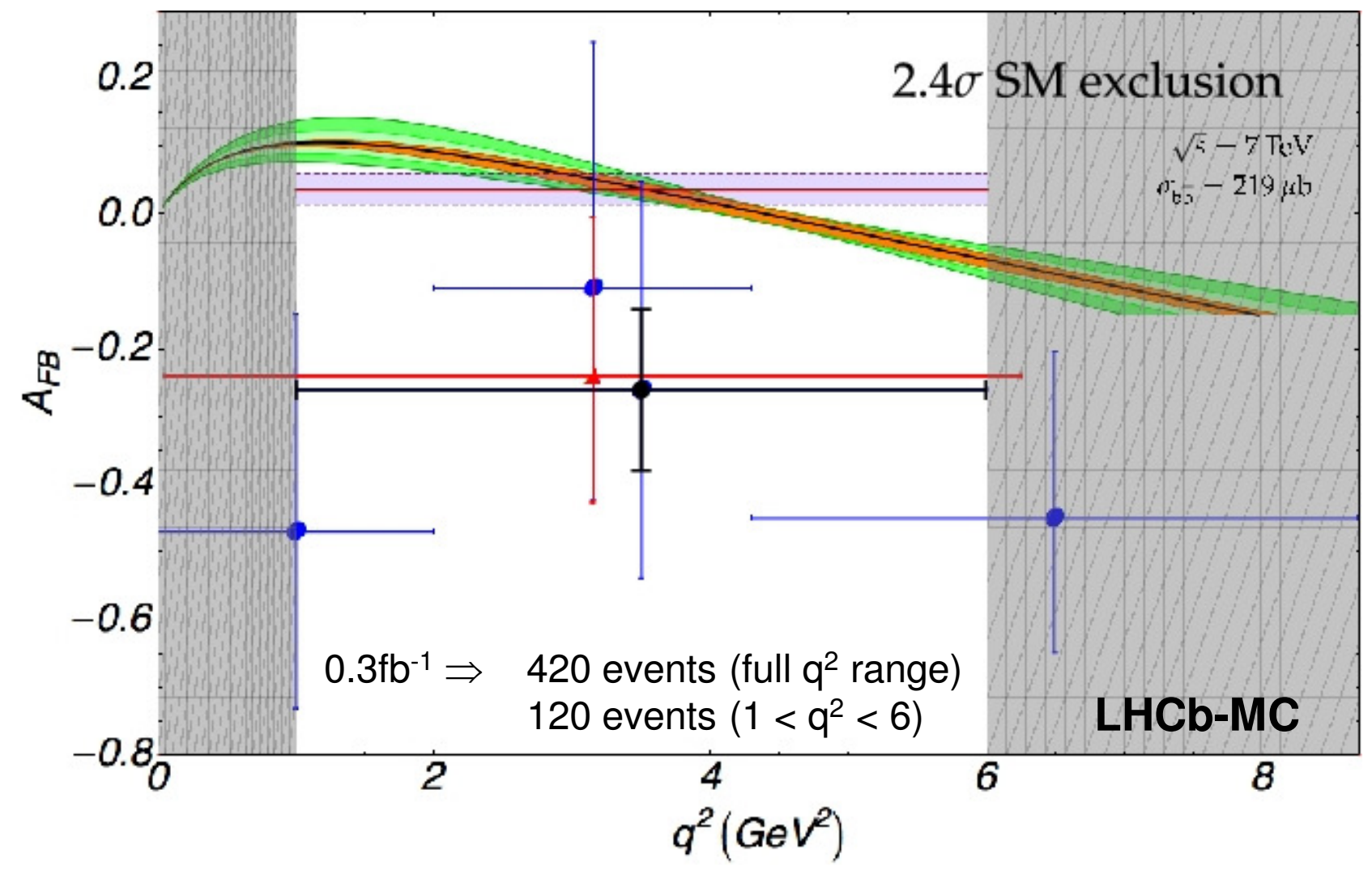




\section{$\mathrm{B}_{\mathrm{d}} \rightarrow \mathrm{K}^{*} \mu \mu: \mathrm{A}_{\mathrm{FB}}$}

- Full angular fit $\left(\theta_{\mathrm{L}}, \theta_{\mathrm{K}}\right.$ and $\left.\phi\right)$ requires $>2 \mathrm{fb}^{-1}$

- Just use $\theta_{L} \rightarrow$ can still measure $A_{F B}$

- LHCb statistical precision (assume Belle central value)

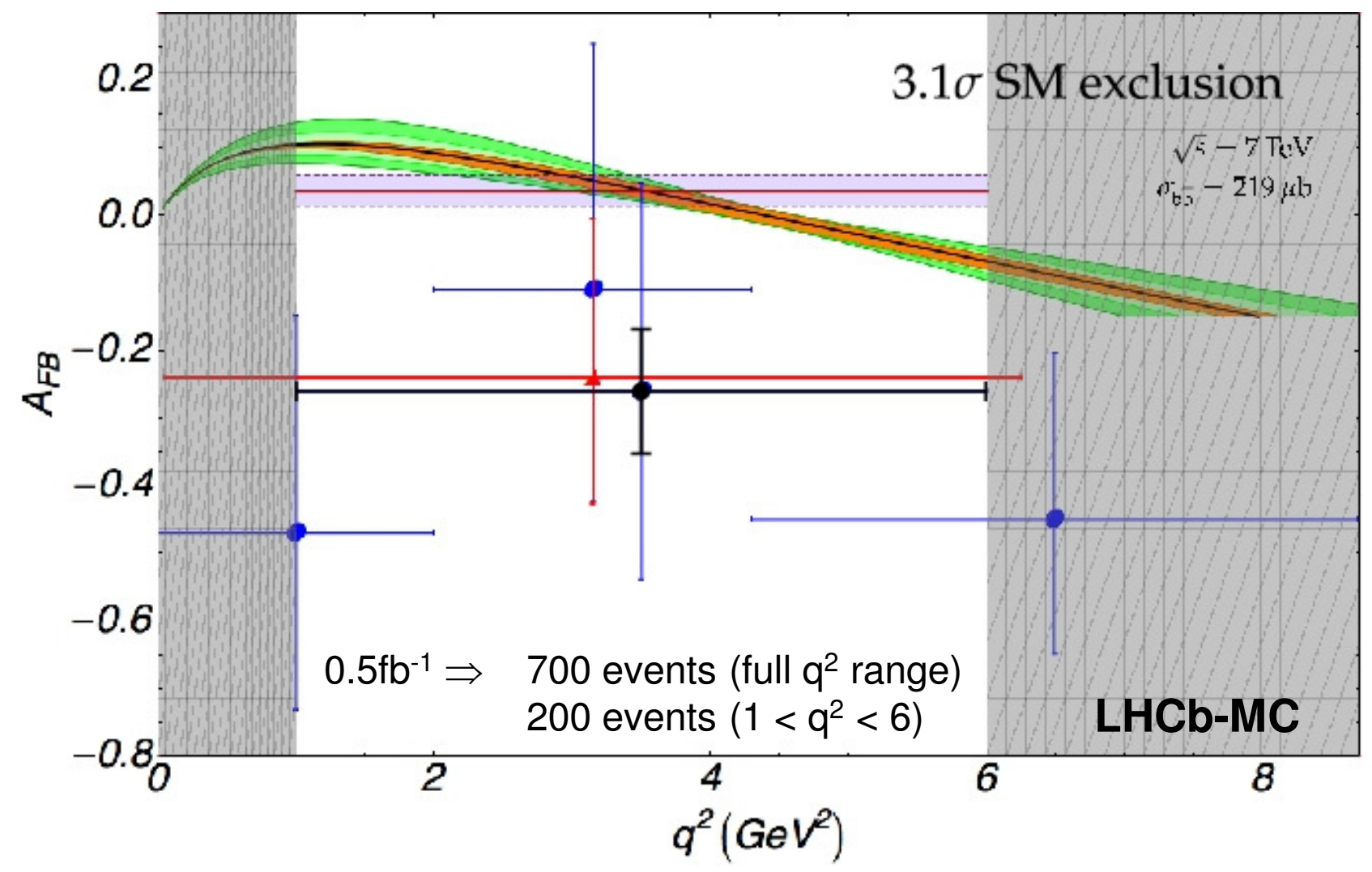




\section{$\mathrm{B}_{\mathrm{s}} \rightarrow \mathrm{J} / \psi \phi$}

$\frac{\mathrm{d}^{4} \Gamma\left(\mathrm{B}_{\mathrm{s}}^{0} \rightarrow \mathrm{J} / \psi \phi\right)}{\mathrm{d} t \mathrm{~d} \cos \theta \mathrm{d} \varphi \mathrm{d} \cos \psi} \equiv \frac{\mathrm{d}^{4} \Gamma}{\mathrm{d} t \mathrm{~d} \Omega} \propto \sum_{k=1}^{6} h_{k}(t) f_{k}(\Omega)$

$\frac{\mathrm{d}^{4} \Gamma\left(\overline{\mathrm{B}}_{\mathrm{s}}^{0} \rightarrow \mathrm{J} / \psi \phi\right)}{\mathrm{d} t \mathrm{~d} \cos \theta \mathrm{d} \varphi \mathrm{d} \cos \psi} \equiv \frac{\mathrm{d}^{4} \bar{\Gamma}}{\mathrm{d} t \mathrm{~d} \Omega} \propto \sum_{k=1}^{6} \overline{h_{k}}(t) f_{k}(\Omega)$

Angular analysis to disentangle the CP-even and CP-odd states

\begin{tabular}{|c|c|c|c|}
\hline$k$ & $h_{k}(t)$ & $\bar{h}_{k}(t)$ & $f_{k}(\theta, \psi, \varphi)$ \\
\hline \hline 1 & $\left|A_{0}(t)\right|^{2}$ & $\left|\bar{A}_{0}(t)\right|^{2}$ & $2 \cos ^{2} \psi\left(1-\sin ^{2} \theta \cos ^{2} \varphi\right)$ \\
2 & $\left|A_{\|}(t)\right|^{2}$ & $\left|\bar{A}_{\| \mid}(t)\right|^{2}$ & $\sin ^{2} \psi\left(1-\sin ^{2} \theta \sin ^{2} \varphi\right)$ \\
3 & $\left|A_{\perp}(t)\right|^{2}$ & $\left|\bar{A}_{\perp}(t)\right|^{2}$ & $\sin ^{2} \psi \sin ^{2} \theta$ \\
4 & $\Im\left\{A_{\|}^{*}(t) A_{\perp}(t)\right\}$ & $\Im\left\{\bar{A}_{\|}^{*}(t) \bar{A}_{\perp}(t)\right\}$ & $-\sin ^{2} \psi \sin 2 \theta \sin \varphi$ \\
5 & $\Re\left\{A_{0}^{*}(t) A_{\|}(t)\right\}$ & $\Re\left\{\bar{A}_{0}^{*}(t) \bar{A}_{\|}(t)\right\}$ & $\frac{1}{\sqrt{2}} \sin 2 \psi \sin { }^{2} \theta \sin 2 \varphi$ \\
6 & $\Im\left\{A_{0}^{*}(t) A_{\perp}(t)\right\}$ & $\Im\left\{\bar{A}_{0}^{*}(t) \bar{A}_{\perp}(t)\right\}$ & $\frac{1}{\sqrt{2}} \sin 2 \psi \sin 2 \theta \cos \varphi$ \\
\hline
\end{tabular}

